\title{
DYNAMICAL SPIN SUSCEPTIBILITY IN THE TD-LDA AND QSGW APPROXIMATIONS
}

\author{
DOE FINAL REPORT, CONTRACT DE-FG02-06ER46302 \\ AND MARK VAN SCHILFGAARDE AND TAKAO KOTANI
}

\begin{abstract}
This project was aimed at building the transverse dynamical spin susceptibility with the TD-LDA and the recently-developed Quasparticle Self-Consisent Approximations, which determines an optimum quasiparticle picture in a self-consistent manner within the $G W$ approximation.

Our main results were published into two papers, (J. Phys. Cond. Matt. 20, 95214 (2008), and Phys. Rev. B83, 060404(R) (2011). In the first paper we present spin wave dispersions for $\mathrm{MnO}, \mathrm{NiO}$, and $\alpha$-MnAs based on quasiparticle self-consistent $G W$ approximation (QSGW). For $\mathrm{MnO}$ and $\mathrm{NiO}, \mathrm{QSG} W$ results are in rather good agreement with experiments, in contrast to the LDA and LDA $+U$ descriptions. For $\alpha$-MnAs, we find a collinear ferromagnetic ground state in QSGW, while this phase is unstable in the LDA.

In the second, we apply TD-LDA to the $\mathrm{CaFeAs}_{2}$ - the first attempt the first $a b$ initio calculation of dynamical susceptibililty in a system with complex electronic structure Magnetic excitations in the striped phase of $\mathrm{CaFe}_{2} \mathrm{As}_{2}$ are studied as a function of local moment amplitude. We find a new kind of excitation: sharp resonances of Stoner-like (itinerant) excitations at energies comparable to the Néel temperature, originating largely from a narrow band of Fe $d$ states near the Fermi level, and coexisting with more conventional (localized) spin waves. Both kinds of excitations can show multiple branches, highlighting the inadequacy of a description based on a localized spin model.

Here we report on the findings of these two papers.
\end{abstract}

\section{INTRODUCTION}

The magnetic linear response is a fundamental property of solids. It is given by the spin susceptibility when the spin-orbit coupling is neglected (as we will do in this article). The spin susceptibility is equivalent with the spin fluctuations, as can be seen from the fluctuationdissipation theorem. Low-energy spin fluctuations can control some low energy phenomena such as magnetic phase transitions, and contribute to resistivity through spin-flip scattering of electrons. Antiferro(AF)-magnetic spin-fluctuations can play an important role in high- $T_{\mathrm{c}}$ superconductors $[1,2]$. It is also the central quantity entering into the description of quantumcritical phenomena $[3,4]$. We expect that reliable first-principle methods to calculate the spin susceptibility should give important clues to understand these phenomena. In this paper, we concentrate on the magnetically-ordered systems, where the spin susceptibility should be dominated by spin waves (SW) at low energy.

In spite of the recent development of such methods, we still have large class of systems where we can hardly calculate the spin susceptibility, e.g, as discussed in Ref.[5]. A typical example is $\mathrm{MnO}$; Solovyev and Terakura gave an analysis for the calculation of its $\mathrm{SW}$ energies [6]. Then they showed main problem is in the non-interacting one-body Hamiltonian $H^{0}$ from which we calculate the non-interacting spin susceptibility used for the calculation of the SW energies. $H^{0}$ given by the local density approximation (LDA), LDA $+U$, or even the optimized effective potential (OEP) [7] are not adequate. In the LDA $+U$ case, they traced the error to a misalignment of the $\mathrm{O}(2 p)$ bands relative to the $\mathrm{Mn}(3 d)$ bands. It is impossible to choose 
the $U$ parameter to correct the misalignment, because the $U$ parameter can only control the exchange splitting within $3 d$ bands. A possibility may be adding some other parameter in addition to $U$ so as to correct the misalignment; however, such a procedure including more parameters become less universal. This situation is somehow similar with the case of optical response (dielectric function) calculation for semiconductors, where $H^{0}$ given by LDA is with too small band gap, thus requires some additional correction like scissors operator. Our case for the spin susceptibility for $\mathrm{MnO}$ is rather worse; LDA supplies too problematic $H^{0}$ to be corrected in a simple manner.

Another possibility is to obtain $H^{0}$ by some hybrid functional; it has been shown that it can work as explained below, however, it could be problematic from the view of universality. Muscat, Wander, and Harrison claimed that a functional B3LYP [8, 9] (containing 20\% of Fock exchange) works even for solids. However, Franchini, Bayer, Podloucky, Paier and Kresse [10] showed that a little different functional, PBE0 is better than B3LYP in order to obtain better agreement with experiments as for the exchange interaction. PBE0 is a combination of $25 \%$ of the Fock exchange with a generalized-gradient approximation(GGA) [11]. However, such a functional could be not so universal, mainly because the effect of screening (therefore the ratio of the Fock exchange) are dependent on materials. In fact, de P. R. Moreira, Illas, and Martin [12] reported that a hybrid functional containing 35\% of Fock exchange gives best results for $\mathrm{NiO}$; the ratio of the Fock exchange is rather different from the case of $\mathrm{MnO}$ by Franchini et al. This is somehow consistent with latest careful examinations by Fuchs Furthmuller, Bechstedt, Shishkin and Kresse [13] and Paier, Marsman, and Kresse [14]; they clarify the fact that a hybrid functional should be limited, because the screening effects (corresponding to the ratio of the Fock exchange) can be material-dependent. These seem to indicate a difficulty to pick up an universally-applicable hybrid functional. This difficulty becomes more problematic when we treat inhomogeneous systems, e.g, to treat the Schotkey-barrier problem, where the screening effects are very different in metal side and in semiconductor side.

Considering these facts, it is necessary to start from good $H^{0}$ without such problems. Our recently-developed quasiparticle self-consistent $G W$ method (QSGW), which includes the above screened exchange effects in a satisfactory manner $[15,16,17,18,19,20,21]$. QSGW determines a reference system of $H^{0}$ representing optimum quasiparticle (QP) picture in the sense of Landau-Silin Fermi liquid theory. As discussed in Ref.[19], it is based on a selfconsistent perturbation theory within all-electron full-potential $G W$ approximation $(G W \mathrm{~A})$, but it is conceptually very different from the usual full self-consistent $G W$. QSGW selfconsistently determines not only $H^{0}$, but also the screened interaction $W$, and the Green's function $G$ simultaneously.

We have shown that QSGW gives QP energies, spin moments, dielectric functions and so on in good agreement with experiments for wide range of materials. There are systematic but a little disagreement from experiments. For example, as shown in Fig.1 in Ref.[17], we see error that calculated band gaps are systematically larger than those by experiments. A recent development by Shishkin, Marsman and Kresse [21] confirmed our conjecture [17] that the inclusion of electron-hole correlation effect in $W$ will correct the error. Their method is a simplified version of the full Bethe-Salpeter equation (BSE) for $W$; it includes only the static and spatially-local part of the first-order term in the BSE, based on the procedure given by Sottile, Olevano, and Reining [22]. However, since the band-gap error itself is small enough, such simplifications may cause no problem. Considering this success, we believe that QSGW is a basis for future development of the electronic structure calculations.

In this paper, we treat $\mathrm{MnO}$ and $\mathrm{NiO}$ with $\mathrm{AF}$ ordering II (AF-II) [23], and $\alpha$-MnAs. $\alpha$ MnAs is NiAs-type grown on GaAs epitaxially, thus is a candidate for spintronics applications 
[24]. For this purpose, we have developed a procedure to calculate the spin susceptibility at zero temperature. It is a general procedure for a given self-consistent method which determines $H^{0}$, even when $H^{0}$ contains non-local potentials as in the Hartree-Fock method. We then apply it to LDA, and to QSGW. After we explain the method in the next section, we will show SW energies obtained with QSGW are in good agreement with experiments for $\mathrm{MnO}$ and NiO. See Ref.[19] for dielectric functions for $\mathrm{NiO}$ and $\mathrm{MnO}$. For MnAs, our calculation shows that a collinear FM ground state is stable in QSGW though it does not in LDA.

At the end of introduction, we give a discussion to justify using the one-particle picture (band picture) of "Mott insulator" for $\mathrm{MnO}$ and $\mathrm{NiO}$; it is essentially given by Terakura, Williams, Oguchi, and Kübler in 1984 [23] (in the following discussion, "charge transfer type" or "Mott type" does not matter). Based on the one-particle picture, the existence of some spin moment (or exchange splitting, equivalently) at each cation site is very essential to make the system insulator. This is consistent with the experimental facts that all the established "Mott insulator" are accompanied with the AF (or some) magnetic ordering. Thus the concept "Mott insulator vs Band insulator" often referred to is misleading, or rather confusing. In order to keep the system insulating, any ordering of spin moment is possible provided the system retains a sufficiently large enough exchange splitting at each site (we need to use the noncollinear mean-field method). In this picture, metal-insulator transition at zero-temperature (e.g, consider a case to compress $\mathrm{NiO}$ ) is nothing but the first-order transition from magneticphase to the non-magnetic phase described by a band picture. On the other hand, the transition at finite temperature to para-magnetic phase occurs because of the entropy effects due to the accumulation of SWs; then the transition is not accompanied with the metalinsulator transition because the exchange splitting (or local moment) at each site is kept even above the Néel temperature $T_{\mathrm{N}}$. This picture is very different from that assumed in Refs. $[25,5]$, where they emphasize the priority of their method LDA $+U+$ "dynamical mean field theory (DMFT)". On the contrary to their claim, we insists that our treatment should be prior and much closer to reality for such systems, because of the following reasons.

i) One-particle treatment in our QSGW allows us to perform parameter-free accurate calculations where we treat all the electrons on the same footing; this is very critical because of the relative position of cation $3 d$ bands to $\mathrm{O}(2 p)$ is important (also their hybridization; we have no SW dispersion without hybridization). Further, we are free from uncontrollable double-counting problem [26], nor the parameter like $U$ which is externally introduced by hand. In contrast, $\mathrm{LDA}+U+\mathrm{DMFT}$ carries these same problems which are in LDA $+U$, or rather highly tangled. Thus it is better to take a calculation by LDA $+U+D M F T$ as a model in cases. As an example, we guess that the distribution probability of the number of $5 f$ electrons in $\delta$-Plutonium calculated by $\mathrm{LDA}+U+\mathrm{DMFT}$ [27] will be easily changed if we shift the relative position (and hybridization) of $5 f$ band with respect to other bands.

ii) The DMFT at zero temperature takes into account the quantum-mechanical onsite fluctuation which is not included within the one-particle picture; it allows a system to be an insulator without magnetic order. However, we expect that such quantum-mechanical fluctuation is not essentially important to determine its ground state for materials like $\mathrm{NiO}$ and $\mathrm{MnO}$. This is based on our findings that QSGW results can well reproduce the optical response [19, 15], and also the magnetic responses as shown in this paper. These QSGW results are not perfect, however, supplies us a good enough starting point. For example, in order to describe the d-d multiplet intra transitions (e.g, see Fig.6 
of Ref.[28] by Fujimori and Minami; they are very weak in comparison with interband transitions), it may be easier to start from the cluster models or so; however, parameters used in these models will be determined by QSGWeven in such a case.

iii) At finite temperature, the DMFT can take into account not only such quantum-mechanical fluctuations, but also the onsite thermal fluctuations simultaneously; this is an advantage of DMFT. However, in $\mathrm{MnO}$ and $\mathrm{NiO}$, low-energy primary fluctuations are limited to the transverse spin fluctuations except phonons. These can be included in DMFT but it is essentially described by the local-moment-disorder [29] as the thermal average of the one-particle picture. Thus no advantage of DMFT if only the thermal fluctuations are important.

\section{METHOD FOR SPIN SUSCEPTIBILITY CALCULATION}

We may divide first-principle methods to calculate SW energies into three classes; (A), (B), and $(\mathrm{C})$. (A) is from the Heisenberg Hamiltonian, whose exchange parameters $J$ are determined from the total energy differences of a set of different spin configurations [30, 31, 32]. (B) and $(\mathrm{C})$ are based on perturbation. (B) estimates $J$ from static infinitesimal spin rotations [33, 34]. We go through the Heisenberg model even in (B). In contrast, (C) determines SW energies directly from the poles in the transverse spin susceptibility $\chi^{+-}\left(\mathbf{r}, \mathbf{r}^{\prime}, t-t^{\prime}\right)$ (defined below) in the random phase approximation (RPA) or time-dependent LDA (TDLDA) [35, 36, 37]. (C) gives the spectrum including life time, and spin-flip excitations. Because $(\mathrm{C})$ is technically difficult, (A) or (B) have been mainly used. (B) is regarded as a simplification of (C); but real implementations entail further approximations.

Our method belongs to (C). Our formalism is applicable to any $H^{0}$ even if it contains non-local potential. At the beginning, we introduce some notations to treat the time-ordered transverse spin susceptibility

$$
\chi^{+-}\left(\mathbf{r}, \mathbf{r}^{\prime}, t-t^{\prime}\right)=-i\left\langle T\left(\hat{S}^{+}(\mathbf{r}, t) \hat{S}^{-}\left(\mathbf{r}^{\prime}, t^{\prime}\right)\right)\right\rangle .
$$

$\langle\ldots\rangle$ denotes the expectation value for the ground state; $T(\ldots)$ means time-ordering, and $\hat{S}^{ \pm}(\mathbf{r}, t)=\hat{S}^{x}(\mathbf{r}, t) \pm i \hat{S}^{y}(\mathbf{r}, t)$ are the Heisenberg operators of spin density. Since we assume collinear magnetic ordering for the ground state, we have $\left\langle\hat{S}^{x}(\mathbf{r}, t)\right\rangle=\left\langle\hat{S}^{y}(\mathbf{r}, t)\right\rangle=0$; $2\left\langle\hat{S}^{z}(\mathbf{r}, t)\right\rangle=M(\mathbf{r})=n^{\uparrow}(\mathbf{r})-n^{\downarrow}(\mathbf{r}) . \quad n^{\uparrow}(\mathbf{r})$ and $n^{\downarrow}(\mathbf{r})$ mean up and down electron densities. $M_{a}(\mathbf{r})$ is the component of $M(\mathbf{r})$ on the the magnetic sites $a$ in unit cell. The Fourier transform of $\chi^{+-}$is

$$
\chi^{+-}\left(\mathbf{T}+\mathbf{r}, \mathbf{r}^{\prime}, \omega\right)=\frac{1}{N} \sum_{\mathbf{q}} e^{i \mathbf{q} \mathbf{T}} \chi_{\mathbf{q}}^{+-}\left(\mathbf{r}, \mathbf{r}^{\prime}, \omega\right),
$$

where $\mathbf{T}$ is a lattice translation vector, and $N$ the number of sites. $\mathbf{r}, \mathbf{r}^{\prime}$ are limited to a unit cell.

Next we derive two conditions Eq. (4) and Eq. (5) below, which $\chi^{+-}$rigorously satisfies. Taking the time derivative of Eq. (1), we obtain

$$
\begin{aligned}
& \frac{\partial}{\partial t^{\prime}} \int d^{3} r^{\prime} \chi^{+-}\left(\mathbf{r}^{\prime}, \mathbf{r}, t^{\prime}-t\right) \\
& =\int d^{3} r^{\prime}\left\langle T\left(\left[\left[\hat{H}, \hat{S}^{+}\left(\mathbf{r}^{\prime}, t^{\prime}\right)\right], \hat{S}^{-}(\mathbf{r}, t)\right]\right)\right\rangle-i \int d^{3} r^{\prime}\left\langle\left[\hat{S}^{+}\left(\mathbf{r}^{\prime}, t^{\prime}\right), \hat{S}^{-}(\mathbf{r}, t)\right]\right\rangle \delta\left(t^{\prime}-t\right),
\end{aligned}
$$


where $[A, B]=A B-B A$. $\hat{H}$ denotes the total Hamiltonian of the system. We have used $\frac{\partial \hat{S}^{+}\left(\mathbf{r}^{\prime}, t^{\prime}\right)}{\partial t^{\prime}}=i\left[\hat{H}, \hat{S}^{+}\left(\mathbf{r}^{\prime}, t^{\prime}\right)\right]$. We assume $\hat{H}$ has rotational symmetry in spin space, so that $\left[\hat{H}, \int d^{3} r^{\prime} \hat{S}^{+}\left(\mathbf{r}^{\prime}, t^{\prime}\right)\right]=0$. Then the first term in the right-hand side is zero. The second term reduces to $M(\mathbf{r})$ because $\left[\hat{S}^{+}\left(\mathbf{r}^{\prime}, t\right), \hat{S}^{-}(\mathbf{r}, t)\right]=2 \hat{S}^{z}(\mathbf{r}, t) \delta\left(\mathbf{r}-\mathbf{r}^{\prime}\right)$. Thus Eq. (3) is reduced to be

$$
\int_{\Omega} d^{3} r^{\prime} \chi_{\mathbf{q}=0}^{+-}\left(\mathbf{r}^{\prime}, \mathbf{r}, \omega\right)=\frac{M(\mathbf{r})}{\omega},
$$

where $\Omega$ denotes the unit-cell volume. Note that Eq. (4) is satisfied for any $\omega$. At $\omega \rightarrow 0$, this means that $M(\mathbf{r})$ is the eigenfunction of $\chi_{\mathbf{q}=0}^{+-}\left(\mathbf{r}, \mathbf{r}^{\prime}, \omega\right)$ with divergent eigenvalue; this is because a magnetic ground state is degenerate for homogeneous spin rotation. Another condition is the asymptotic behavior as $\omega \rightarrow \infty$. It is given as

$$
\chi^{+-}\left(\mathbf{r}^{\prime}, \mathbf{r}, \omega\right) \rightarrow \frac{M(\mathbf{r})}{\omega} \delta\left(\mathbf{r}-\mathbf{r}^{\prime}\right)+O\left(1 / \omega^{2}\right) .
$$

This can be easily derived from the spectrum representation of $\chi^{+-}$. We use Eq. (4) and Eq. (5) to determine the effective interaction $\bar{U}$ in the following.

As in Ref. [38], we define the effective interaction $U\left(\mathbf{r}, \mathbf{r}^{\prime}, \omega\right)$ as the difference between $\left(\chi^{+-}\right)^{-1}$ and the non-interacting counterpart: $\left(\chi^{0+-}\right)^{-1}\left(\mathbf{r}, \mathbf{r}^{\prime}, \omega\right)$;

$$
\left(\chi^{+-}\right)^{-1}=\left(\chi^{0+-}\right)^{-1}+U
$$

In TDLDA, $U$ is the second derivative of the exchange-correlation energy, $U\left(\mathbf{r}, \mathbf{r}^{\prime}\right)=-\delta^{2} E_{\mathrm{xc}} / \delta S^{+}(\mathbf{r}) \delta S^{-}\left(\mathbf{r}^{\prime}\right)=$ $I_{\mathrm{xc}}(\mathbf{r}) \delta\left(\mathbf{r}-\mathbf{r}^{\prime}\right)$, which is local $U\left(\mathbf{r}, \mathbf{r}^{\prime}\right) \propto \delta\left(\mathbf{r}-\mathbf{r}^{\prime}\right), \omega$-independent, and positive. Then we can show that $\chi^{+-}$in TDLDA satisfies conditions Eq. (4) and Eq. (5) automatically [39]. In the case of $H^{0}$ containing nonlocal potentials (e.g. in the case of the Hartree-Fock method), $U$ is no longer independent of $\omega$. This is because the natural expansion of $\chi^{+-}$in the many-body perturbation theory requires solving the Bethe-Salpeter Eq. for the two-body propagator $\chi^{+-}\left(\mathbf{r}_{1}, \mathbf{r}_{2} ; \mathbf{r}_{3}, \mathbf{r}_{4}, \omega\right)$, thus $U$ defined in Eq. (6) is not directly identified as a kinds of diagrams. Ref.[37] did not pay attention to this point. We can calculate $\chi^{0+-}$ in Eq. (6) as

$$
\begin{aligned}
& \chi_{\mathbf{q}}^{0+-}\left(\mathbf{r}, \mathbf{r}^{\prime}, \omega\right) \quad=\sum_{\mathbf{k} n \downarrow}^{\text {occ }} \sum_{\mathbf{k}^{\prime} n^{\prime} \uparrow}^{\text {unocc }} \frac{\Psi_{\mathbf{k} n \downarrow}^{*}(\mathbf{r}) \Psi_{\mathbf{k}^{\prime} n^{\prime} \uparrow}(\mathbf{r}) \Psi_{\mathbf{k}^{\prime} n^{\prime} \uparrow}^{*}\left(\mathbf{r}^{\prime}\right) \Psi_{\mathbf{k} n \downarrow}\left(\mathbf{r}^{\prime}\right)}{\omega-\left(\epsilon_{\mathbf{k}^{\prime} n^{\prime} \uparrow}-\epsilon_{\mathbf{k} n \downarrow}\right)+i \delta} \\
& +\sum_{\mathbf{k} n \downarrow}^{\text {unocc }} \sum_{\mathbf{k}^{\prime} n^{\prime} \uparrow}^{\text {occ }} \frac{\Psi_{\mathbf{k} n \downarrow}^{*}(\mathbf{r}) \Psi_{\mathbf{k}^{\prime} n^{\prime} \uparrow}(\mathbf{r}) \Psi_{\mathbf{k}^{\prime} n^{\prime} \uparrow}^{*}\left(\mathbf{r}^{\prime}\right) \Psi_{\mathbf{k} n \downarrow}\left(\mathbf{r}^{\prime}\right)}{-\omega-\left(\epsilon_{\mathbf{k} n \downarrow}-\epsilon_{\mathbf{k}^{\prime} n^{\prime} \uparrow}\right)+i \delta},
\end{aligned}
$$

where $\mathbf{k}^{\prime}=\mathbf{q}+\mathbf{k} \cdot \chi^{0+-}\left(\mathbf{r}, \mathbf{r}^{\prime}, \omega=0\right)$ is negative definite matrix. Our definition of $\chi^{+-}$and also $\chi^{0+-}$ can be different in sign from other definitions in the literature because we start from Eq. (1).

In order to realize an efficient computational method, we assume that the magnetization is confined to magnetic atomic sites, and we explicitly treat only a degree of freedom of spin rotation per each site. Then we can determine $U$ with the help of Eq. (4) and Eq. (5) as in the following. As a choice to extract the degrees of freedom, we consider a matrix $D(\mathbf{q}, \omega)$ as

$$
(D(\mathbf{q}, \omega))_{a a^{\prime}}=\int_{a} d^{3} r \int_{a^{\prime}} d^{3} r^{\prime} \bar{e}_{a}(\mathbf{r}) \chi_{\mathbf{q}}^{+-}\left(\mathbf{r}, \mathbf{r}^{\prime}, \omega\right) \bar{e}_{a^{\prime}}\left(\mathbf{r}^{\prime}\right)
$$

and $D^{0}(\mathbf{q}, \omega)$ defined in the same manner. The dimension of the matrix $D(\mathbf{q}, \omega)$ is the number of magnetic sites. Here we define $e_{a}(\mathbf{r})=M_{a}(\mathbf{r}) / M_{a}$ where $M_{a}=\int_{a} d^{3} r M_{a}(\mathbf{r})$; and 
define $\bar{e}_{a}(\mathbf{r})$ so that $\bar{e}_{a}(\mathbf{r}) \propto e_{a}(\mathbf{r})$ and $\int d^{3} r \bar{e}_{a}(\mathbf{r}) e_{a}(\mathbf{r})=1$; thus $\bar{e}_{a}(\mathbf{r})=e_{a}(\mathbf{r}) / \int_{a} d^{3} r\left(e_{a}(\mathbf{r})\right)^{2}$. Corresponding to Eq. (6), we define the effective interaction $(\bar{U}(\mathbf{q}, \omega))_{a a^{\prime}}$ as

$$
(D(\mathbf{q}, \omega))^{-1}=\left(D^{0}(\mathbf{q}, \omega)\right)^{-1}+\bar{U}(\mathbf{q}, \omega) .
$$

For the calculation of $D^{0}(\mathbf{q}, \omega)$ from Eq. (7), we use the tetrahedron technique [19], which allow us to use fewer $\mathbf{k}$ points in the first Brillouin zone (BZ) than those required for the sampling method [37]. $\bar{U}$ defined in Eq. (9) should include all the downfolded contributions from all the other degrees of freedom. We now assume that $\bar{U}$ is q-independent and sitediagonal, so that it can be written as $\bar{U}_{a a^{\prime}}(\mathbf{q}, \omega)=U_{a}(\omega) \delta_{a a^{\prime}}$. Since Eq. (4) reduces to a constraint $\sum_{a^{\prime}}(D(\mathbf{q}=0, \omega))_{a^{\prime} a}=M_{a} / \omega$, we determine $\bar{U}_{a}(\omega)$ from

$$
\bar{U}_{a}(\omega)=\frac{\omega}{M_{a}} \delta_{a a^{\prime}}-\left(\frac{\sum_{b} M_{b}\left(D^{0}(\mathbf{q}=0, \omega)\right)_{b a}^{-1}}{M_{a}}\right) \delta_{a a^{\prime}} .
$$

With this $\bar{U}_{a}(\omega)$ for Eq. (9), we finally have

$$
\begin{aligned}
& (D(\mathbf{q}, \omega))^{-1}=\frac{\omega}{M_{a}} \delta_{a a^{\prime}}-\bar{J}(\mathbf{q}, \omega) \\
& \bar{J}(\mathbf{q}, \omega)=-\left(D^{0}(\mathbf{q}, \omega)\right)^{-1}+\left(\frac{\sum_{b} M_{b}\left(D^{0}(\mathbf{q}=0, \omega)\right)_{b a}^{-1}}{M_{a}}\right) \delta_{a a^{\prime}} .
\end{aligned}
$$

Eq. (5) reduces to $(D(\mathbf{q}, \omega))_{a^{\prime} a}^{-1} \rightarrow \frac{\omega}{M_{a}} \delta_{a a^{\prime}}$ at $\omega \rightarrow \infty ;(D(\mathbf{q}, \omega))_{a^{\prime} a}^{-1}$ given by Eq. (10) gives this correct asymptotic behavior. Note that we determine $U$ just from the requirement Eq. (4) because of our approximations "onsite only $U$ " and "a basis per magnetic site". If we need to go beyond such approximation (e.g. multiple basis per site), it will be necessary to introduce additional informations, e.g. a part of $\chi(\mathbf{q}, \omega=0)$ evaluated by numerical linear-response calculations (perform the QSGW self-consistent calculations with bias fields). By Fourier transformation, we can transform $(D(\mathbf{q}, \omega))_{a^{\prime} a}$ into $D_{R R^{\prime}}(\omega)$; the same is also for $D^{0}, J$ and so on. Here $R=\mathbf{T} a$ is the composite index to specify an atom in the crystal. For later discussion we define

$$
J(\mathbf{q}, \omega)=-\left(D^{0}(\mathbf{q}, \omega)\right)^{-1}+\frac{\delta_{a a^{\prime}}}{D_{a a}^{0}(\omega)},
$$

where $D_{a a}^{0}(\omega)$ is shorthand for $D_{\mathbf{T} a \mathbf{T} a}^{0}(\omega)$; it is $\mathbf{T}$ independent. The second term in Eq. (12) is included just in order to remove the onsite term from $J$. Then Eq. (11) can be written as

$$
\bar{J}(\mathbf{q}, \omega)=J(\mathbf{q}, \omega)-\left(\frac{\sum_{b} M_{b} J_{b a}(\mathbf{q}=0, \omega)}{M_{a}}\right) \delta_{a a^{\prime}} .
$$

Here, the second term (onsite term) in Eq. (12) is irrelevant because of the cancellation between two terms in Eq. (13).

The preceding development for $(D(\mathbf{q}, \omega))^{-1}$ facilitates a comparison with the Heisenberg model, whose Hamiltonian is $\mathcal{H}=-\sum_{R} \sum_{R^{\prime}} J_{R R^{\prime}}^{\mathcal{H}} \mathbf{S}_{R} \cdot \mathbf{S}_{R^{\prime}}(R=\mathbf{T} a)$. As shown in Appendix A, the inverse of the susceptibility in the Heisenberg model is:

$$
\left(D^{\mathcal{H}}(\mathbf{q}, \omega)\right)^{-1}=\frac{\omega}{M_{a}} \delta_{a a^{\prime}}-\bar{J}^{\mathcal{H}}(\mathbf{q})
$$

where $M_{a}=\left|2 \mathbf{S}_{R}\right|$. Let us compare Eq. (14) with Eq. (10). This $\bar{J}_{a a^{\prime}}^{\mathcal{H}}(\mathbf{q})$ is given by Eq. (25), which is almost the same as Eq. (13); only the difference is whether we use $J^{\mathcal{H}}$ or $J$. This suggests how to construct the Heisenberg model which reproduces Eq. (10) as good as possible; a possibility is that we simply assign $J(\mathbf{q}, \omega=0)$ (neglecting the $\omega$-dependence) as $J^{\mathcal{H}}(\mathbf{q})$. We 
have confirmed that this approximation is good enough to reproduce SW energies in the case for $\mathrm{MnO}$ and $\mathrm{NiO}$. However, it is not true in the case of $\alpha$-MnAs; then we have used another procedure given by Katsnelson and Lichtenstein [39]: we identify $J(\mathbf{q}, \omega=($ SW energy at $\mathbf{q})$ ) as $J^{\mathcal{H}}$. This construction exactly reproduces $\mathrm{SW}$ energies calculated from $D^{\mathbf{q}, \omega}$.

As a further approximation to calculate $J(\mathbf{q}, \omega=0)$, we can expand it in real space as (omit $\omega$ for simplicity)

$$
-J_{R R^{\prime}}=\left(D_{R R^{\prime}}^{0}\right)^{-1}-\frac{\delta_{R R^{\prime}}}{D_{R}^{0}}=\left(D_{R}^{0} \delta_{R R^{\prime}}+D_{R R^{\prime}}^{0, \text { off }}\right)^{-1}-\frac{\delta_{R R^{\prime}}}{D_{R}^{0}} \approx \frac{1}{D_{R}^{0}} D_{R R^{\prime}}^{0, \text { off }} \frac{1}{D_{R^{\prime}}^{0}},
$$

where we use Eq. (12); we use notation that onsite part $D_{R}^{0}=D_{R R}^{0}$ and the off-site part $D_{R R^{\prime}}^{0 \text { off }}=D_{R R^{\prime}}^{0}-D_{R R^{\prime}}^{0} \delta_{R R^{\prime}}$. Here we have used the assumption that $D_{R R^{\prime}}^{\text {off }}$ are small in comparison with onsite term $D_{R}^{0}$. This approximation corresponds to the usual second-order perturbation scheme of the total energy; if the spin rotation perfectly follows the rotation of the one-particle potential, $\frac{1}{D_{R}^{0}}$ is trivial; it is equal to the difference of the one-particle potential between spins (exchange-correlation potential in the case of DF) because $\frac{1}{D_{R}^{0}}$ is the inverse linear-response to determine the one-particle potential for given spin rotation. Essentially the same equation as Eq. (15) was used in Refs. [33, 34]. In cases, this approximation is somehow mixed up with the "long wave approximation" to expanding $J$ around $D^{0}(\mathbf{q}=0)$ [38]; however, they should be differentiated. In order to have rough estimate of $J_{R R^{\prime}}$, we can further reduce this to the two sites model as originally presented by Anderson and Hasegawa [40, 41]. For an AF magnetic pair (half-filled case), we obtain the following estimate:

$$
J_{R R^{\prime}} \approx-\frac{1}{D_{R}} D_{R R^{\prime}}^{0, \mathrm{off}} \frac{1}{D_{R^{\prime}}} \sim-\frac{4 t^{2}}{\Delta E_{\mathrm{ex}} M},
$$

where $t$ denotes the transfer integral, and $\Delta E_{\mathrm{ex}}$ is the onsite exchange splitting. We have used $D_{R} \sim \frac{M}{\Delta E_{\mathrm{ex}}}$, and $D_{R R^{\prime}} \sim \frac{M}{\Delta E_{\mathrm{ex}}} \times\left(\frac{2 t}{\Delta E_{\mathrm{ex}}}\right)^{2}$.

Some additional comments. Our formalism here is not applicable to the non-magnetic systems, where $M(\mathbf{r})=0$ everywhere. Then we need to determine $U$ in other ways. A possibility is utilizing the static numerical linear-response calculations; it gives the information of the static $(\omega=0)$ part of $\chi_{\mathbf{q}}^{+-}$directly (easiest spin-polarization mode at each site). Then it will be possible to determine $U$ from such informations together with some additional assumptions. In the case of systems like Gd where the $d$ shell and $f$ shell can polarize separately, we may need to extend our formulation so as to include non-locality of $U$ (e.g. $U$ can be parametrized as $U_{i j k l}$ where $i, j, k, l$ are atomic eigenfunction basis for $d$ or $f$ channel).

\section{RESULT AND DISCUSSION}

3.1. $\mathrm{MnO}$ and $\mathrm{NiO}$. Fig. 1 shows the calculated $\mathrm{SW}$ energies $\omega(\mathbf{q})$ for $\mathrm{MnO}$ and $\mathrm{NiO}$. (We used $1728 k$-points in the BZ for all calculations, including MnAs.) $\omega(\mathbf{q})$ calculated from the LDA is too large, as earlier workers have found $[6,5]$. The detailed shape of $\omega(\mathbf{q})$ is different from earlier work however: in Ref. [5], peaks in $\omega(\mathbf{q})$ occur near $200 \mathrm{meV}$ for $\mathrm{NiO}$, much lower than what we find. QSGW predicts $\omega(\mathbf{q})$ in good agreement with experimental data.

The difference of results between QSGW and LDA is understood by Eq. (16). $J_{R R^{\prime}}$ between nearest AF sites essentially determine the SW energies (exactly speaking, three $J$ parameters as shown in Table 1). The LDA severely underestimates $\Delta E_{\text {ex }}$. This can be corrected by LDA $+U$, however, Solovyev and Terakura [6] showed that it fails to reproduce SW energies as we mentioned in the introduction. This means that the transfer $t$ is also wrong in LDA $+U$; in fact $t$ is through the hybridization with Oxygen $2 p$ (superexchange). In other words, the 
agreements with SW experiments in QSGW indicates that both of them are well described by QS $G W$. Together with the fact that QS $G W$ showed good agreements with optical experiments $[15,19]$ for $\mathrm{MnO}$ and $\mathrm{NiO}$, we claim that our one-particle picture given by QSGW captures the essence of the physics for these systems. Our claim here is opposite to Refs. [25, 5] where they claimed that the one-particle picture can not capture the essence.
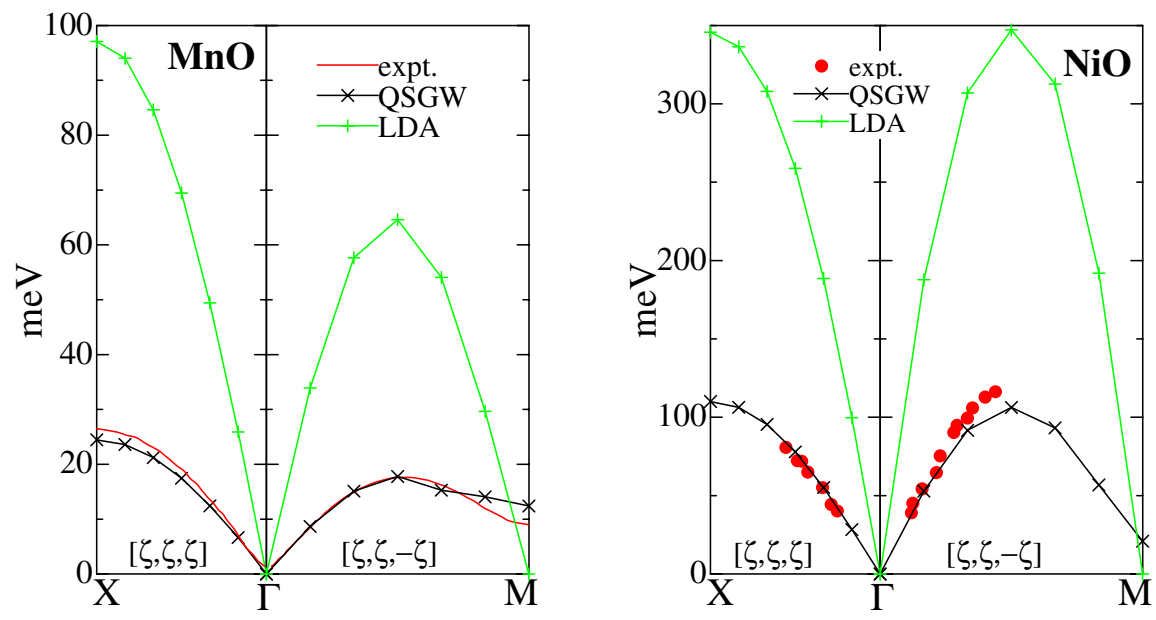

Figure 1. (color online) Spin wave dispersion $\omega(\mathbf{q})$ for $\mathrm{MnO}$ and $\mathrm{NiO}$ calculated from the LDA and QSGW. Solid line without symbols in $\mathrm{MnO}$ or dots in $\mathrm{NiO}$ (red) are experimental values [42, 43]. We used experimental lattice constants 4.55 and $4.17 \AA$ for $\mathrm{MnO}$ and $\mathrm{NiO}$ respectively.

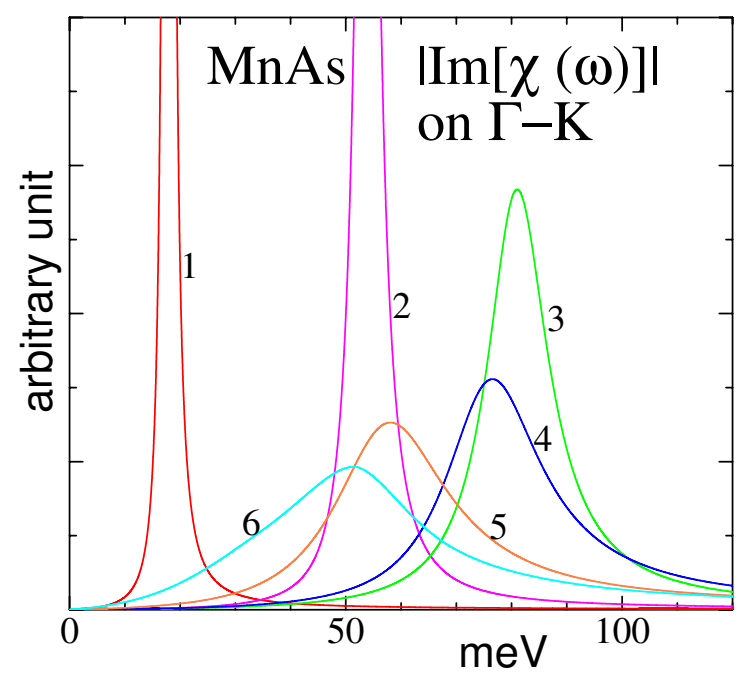

Figure 2. (color online) Im part of $\operatorname{Tr}\left[\chi^{+-}(\mathbf{k}, \omega)\right]$ for QSGW. Data are for 6 $k$-points, all along the $\Gamma-\mathrm{K}$ line. The $k$-point is $i / 6 \mathrm{~K}$ (thus $i=6$ falls at $\mathrm{K}$ ). Peak positions and full-width-half-maxima are shown in the $\Gamma-\mathrm{K}$ line of Fig. 3. 


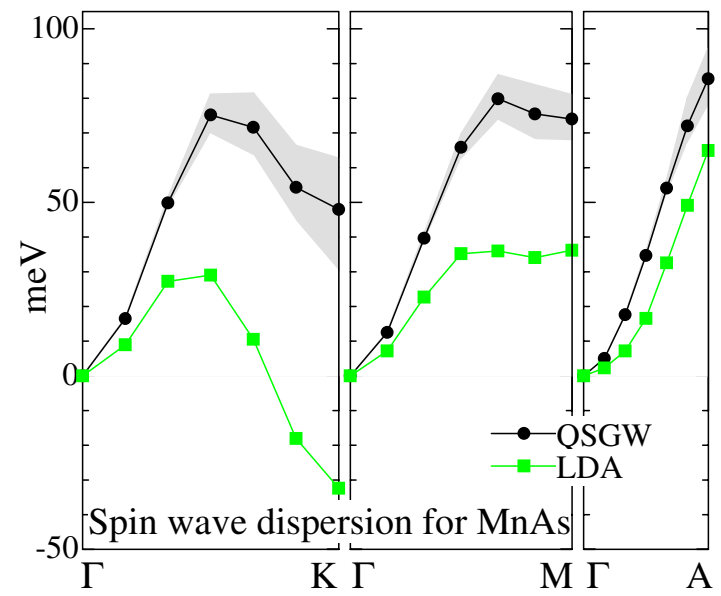

Figure 3. (color online) Spin wave dispersion $\omega(\mathbf{q})$ in $\alpha$-MnAs. QSGW results (circles) are enveloped by hatched regions, which indicate the full-width at half-maximum of the spin wave, and is a measure of the rate of SW decay. LDA (squares) predicts negative SW energies around K; indicating that the collinear FM ground state is not stable. The experimental lattice constants $a=3.70 \AA$ and $c / a=1.54$ were used.

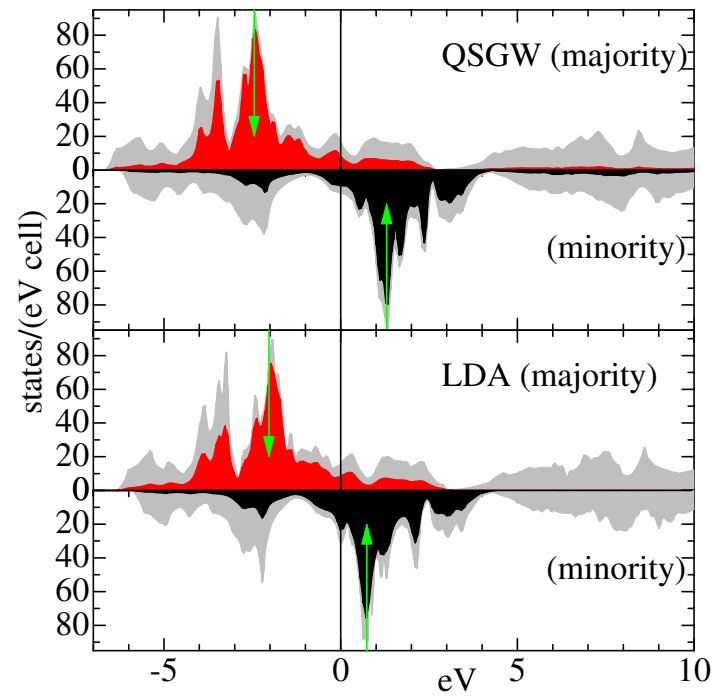

FiguRE 4. (color online) quasiparticle DOS for $\alpha$-MnAs. Lighter hatchings indicate total DOS; darker(black and red) hatchings indicate the partial $d$ contribution, whose centers of gravity are shown by arrows. The Fermi energy is at zero.

3.2. $\alpha$-MnAs. Because $\alpha$-MnAs is observed to be a FM with a moment of $3.4 \mu_{B}[44]$, we construct $H^{0}$ assuming a FM ground state. Inspection of the density of states (DOS) in Fig. 4, shows that QSGW predicts $\Delta E_{\mathrm{ex}} \sim 1.0 \mathrm{eV}$ larger the LDA. This difference is reflected in the spin moment: $M_{a}=3.51 \mu_{B}$ in QSGW, 3.02 $\mu_{B}$ in LDA. Fig. 2 shows the imaginary part of $\operatorname{Tr}\left[\chi^{+-}(\mathbf{q}, \omega)\right]$ along $\Gamma-K$ line. Sharp SW peaks are seen at small $\mathbf{q}$; they broaden with 
increasing q. Fig. 3 shows the peak positions, corresponding to SW energies $\omega(\mathbf{q})$. Hatchmarks indicate the full-width at half-maximum, extracted from data such as that depicted in Fig. 2. This corresponds to the inverse lifetime of a SW which decays into spin-flip excitations. (Our calculation gives no width for $\mathrm{MnO}$ and $\mathrm{NiO}$, because of the large gap for the spin-flip excitations). SW peaks are well identified all the way to the BZ boundary. We find that the collinear FM ground state is not stable in the LDA: as Fig. 3 shows, $\omega(\mathbf{q})<0$ around K. (Among all possible collinear configurations, the FM state may be the most stable. We did not succeed in finding any collinear configuration more stable than the FM one. A similar conclusion was drawn for the PBE GGA functional [31].) On the other hand, QSGW predicts stable collinear ground state, that is, $\omega(\mathbf{q})>0$ everywhere. However, even in QSGW, the SW energies are still low around $\mathrm{K}$, which is a vector that connects nearest neighbor Mn sites in $\mathrm{x}-\mathrm{y}$ plane. If this SW energy is further lowered for some reason, we may have a frustrated spin system because of the triangle (honeycomb) lattice of the Mn sites. This could be related to the anomalous phase diagram of MnAs, which can easily occur through the small changes in lattice structure associated with higher-temperature phases.

We can qualitatively understand the difference of SW energies between QSGW and LDA from the difference of $\Delta E_{\mathrm{ex}}$. Let us consider the energy difference of FM and AFM states for two-site model as illustrated in Ref.[41]. Then the energy gain of a FM pair is independent of $\Delta E_{\text {ex }}$ when some of majority states are occupied (less than half filling); we measure the energy from the majority spin's atomic level as the zero. In contrast, the gain of a AFM pair increases with decreasing $\Delta E_{\text {ex }}$. Overall, the LDA with its smaller $\Delta E_{\text {ex }}$, should contain a stronger AFM tendency.

TABLE 1. Magnetic parameters calculated by QSGW and LDA (in parenthesis). Muffin-tin radii $R$ for cations were taken to be $2.48(\mathrm{MnO}), 2.33$ $(\mathrm{NiO})$, and $2.42(\mathrm{MnAs})$ a.u. $M_{a}$ is the spin moment within the muffin tin. Our approximation is equivalent to the assumption for $U$ as $U\left(\mathbf{r}, \mathbf{r}^{\prime}, \omega\right)=\sum_{a}\left(U_{a}^{0}+\omega U_{a}^{1}+\ldots\right) e_{a}(\mathbf{r}) e_{a}\left(\mathbf{r}^{\prime}\right)$ in Eq. (6). Then $U_{a}^{0}$ is written as $U_{a}^{0}=\int_{a} d^{3} r \int_{a} d^{3} r^{\prime} e_{a}(\mathbf{r}) e_{a}\left(\mathbf{r}^{\prime}\right) U\left(\mathbf{r}, \mathbf{r}^{\prime}, \omega=0\right)$. Exchange parameters $J_{1+}, J_{1-}, J_{2}$ are shown for $\mathrm{MnO}$ and $\mathrm{NiO}$. Total spin moments for MnAs are $7.00 \mu_{B} / \operatorname{cell}(\mathrm{QS} G W)$ and $5.89 \mu_{B} /$ cell (LDA). Our definition of $J_{1+}, J_{1-}, J_{2}$ follows that of Ref. [6], except we distinguish $J_{1+}$ and $J_{1-}[42]$.

\begin{tabular}{lccc} 
& $\mathrm{MnO}$ & $\mathrm{NiO}$ & $\alpha$-MnAs \\
\hline$U_{a}^{0}(\mathrm{eV})$ & $2.43(0.95)$ & $4.91(1.64)$ & $1.08(0.93)$ \\
$M_{a}\left(\mu_{B}\right)$ & $4.61(4.35)$ & $1.71(1.21)$ & $3.51(3.02)$ \\
\hline$J_{1+}(\mathrm{meV})$ & $-2.8(-14.7)$ & $-0.77(0.3)$ & \\
$J_{1-}$ & $-4.8(-14.7)$ & $-1.00(0.3)$ & \\
$J_{2}$ & $-4.7(-20.5)$ & $-14.7(-28.3)$ & \\
\hline $\mathrm{T}_{\mathrm{N} \text { or } \mathrm{T}_{\mathrm{c}}(\mathrm{K})}$ & 111 & 275 & 510 \\
(experiment) & $122^{1}$ & $523^{1}$ & 400
\end{tabular}

3.3. Determined Parameters and related Quantities. Table 1 shows the effective interaction $U_{a}^{0}$ (interaction between unit spins). In $\mathrm{NiO}$ and $\mathrm{MnO}, U_{a}^{0}$ as calculated by LDA is much smaller than the QSGW result. This is because the LDA underestimates bandgaps in $\mathrm{NiO}$ and $\mathrm{MnO}$, thus overestimates the screening. $U_{a}^{0}$ is twice larger in $\mathrm{NiO}$ than in $\mathrm{MnO}$. This is because $M_{a}(\mathbf{r})$ is more localized in NiO; in fact, the QSGW dielectric constants $\epsilon_{\infty}$ are similar $\left(\epsilon_{\infty}=3.8(\mathrm{MnO})\right.$ and $\left.4.3(\mathrm{NiO})[19]\right)$, suggesting that the screened Coulomb interaction 
$U\left(\mathbf{r}, \mathbf{r}^{\prime}\right)$ is similar in the two materials. $U_{a}^{0}$ is smaller in MnAs than in $\mathrm{MnO}$, because it is a metal.

For $\mathrm{MnO}$ and $\mathrm{NiO}$, we confirmed that $J_{R R^{\prime}}$ is non-negligible only for the three nearestneighbors $(\mathrm{NN})$ (Table 1$). J_{1+}$ and $J_{1-}$ refer to 1 st $\mathrm{NN}$, spins parallel and spins antiparallel, respectively. $J_{2}$ refers to 2 nd $\mathrm{NN}[42] . J_{1+}$ and $J_{1-}$ by QSGW are quite different in $\mathrm{MnO}$, while in LDA $J_{1+} \approx J_{1-}$, resulting in $\omega(\mathrm{M}) \approx 0$ in that case.

For MnAs in QSGW, the expansion coefficients written as $\left.\left(M^{-1}\right)_{a a^{\prime}} \equiv \frac{\partial\left(D^{\mathbf{q} \omega}\right)_{a a^{\prime}}^{-1}}{\partial \omega}\right|_{\omega=0}$, is rather dependent on $\mathbf{q}$; nor is $\left(M^{-1}\right)_{a a^{\prime}} \propto \delta_{a a^{\prime}}$. Off-diagonal contributions of $\left(M^{-1}\right)_{a a^{\prime}}$ give $\sim 10 \%$ contribution to SW energies. In addition, its inverse of the diagonal element $1 /\left(M^{-1}\right)_{a a}$ is reduced by $\sim 0.5 \mu_{B}$ at certain points in the BZ. In this case, mapping to a Heisenberg Hamiltonian has less clear physical meaning.

3.4. Calculation of $T_{\mathrm{N}}$ and $T_{\mathrm{c}}$ based on the Heisenberg model. From obtained $J_{R R^{\prime}}$, we estimated $T_{\mathrm{N}}$ ( $T_{\mathrm{c}}$ for MnAs) for QSGW (Table 1) using the cluster variation method adapted to the Heisenberg model [46], which assumes classical dynamics of spins under $\mathcal{H}$. In $\mathrm{NiO}$, the calculated $T_{\mathrm{N}}$ is only $\sim 50 \%$ of experiment. There are two important effects that explain the discrepancy: (a) QSGW overestimates the $d d$ exchange splitting [17, 19], and (b) the classical treatment of quantum dynamics of spins under $\mathcal{H}$. Both effects will increase $T_{\mathrm{N}}$. Considering that QSGW well reproduces SW energies (Fig. 1), the errors connected with (a) would not seem to be so serious in $\mathrm{MnO}$ and $\mathrm{NiO}$. (b) can be rather important, especially when the local moment is small. This is a general problem as discussed in Ref. [5]: Heisenberg parameters that reproduce SW energies well in $\mathrm{NiO}$ do not yield a correspondingly good $T_{\mathrm{N}}$. If we multiply our classical $T_{\mathrm{N}}$ by a factor $S(S+1) / S^{2} \approx 1.86\left(\right.$ as $2 S=M_{a}=1.71$ ), which is the ratio of quantum to classical $T_{\mathrm{N}}$ in mean field theory, we have better agreement with experiment. This is what Hutching et al. used [43]. On the other hand, evaluation of the quantum Heisenberg model using a Green's function technique show that the mean-field theory rather strongly overestimates quantum corrections [47]. Also, $T_{\mathrm{N}}$ is already close to the experimental value in $\mathrm{MnO}$. This is explained in part because correction $(b)$ is less important in $\mathrm{MnO}$, since $S$ is larger. Further, we have large contributions to $T_{\mathrm{N}}$ from $J_{1 \pm}$ in $\mathrm{MnO}$, but not in $\mathrm{NiO}$. Around $T_{\mathrm{N}}, J_{1+}$ and $J_{1-}$ will tend to approach some average value, which reduces $\omega(\mathbf{q})$ and therefore $T_{\mathrm{N}}$ (recall $\omega(\mathrm{M})=0$ when $\left.J_{1+}=J_{1-}\right)$. The temperature-dependence of $J$ is not accounted for here.

$J_{R R^{\prime}}$ exhibits long-ranged, oscillatory behavior in MnAs: its envelope falls off as $\left|\mathbf{R}-\mathbf{R}^{\prime}\right|^{3}$ as predicted by RKKY theory for a metal. Consequently, it is not so meaningful to estimate $T_{\mathrm{c}}$ from just a few NN, as was done recently [31,32]. Shells up to 25th-neighbors are required to converge $T_{\mathrm{c}}$ to within $5 \%$ or so. The calculated $T_{\mathrm{c}}$ is $110 \mathrm{~K}$ too high in comparison with experiment. Taking $(a)$ into account will improve the agreement; however, there are many factors that make a precise calculation very difficult. We also need to take $(b)$ into account; in addition, other factors such as assumptions within the Heisenberg model, may give nonnegligible contributions.

In conclusion, we present a simple method to calculate spin susceptibility, and applied it in the QSGW method. SW energies for $\mathrm{MnO}$ and $\mathrm{NiO}$ are in good agreement with experiments; in $\alpha$-MnAs the FM ground state is stable, which also agrees with experiment (to our knowledge, no SW energies have been published in $\alpha$-MnAs). LDA results come out very differently in each material. By mapping to the Heisenberg model, we estimated $T_{\mathrm{N}}$ or $T_{\mathrm{c}}$. We found some disagreement with experiments, and discussed some possible explanations. 


\section{Appendix A. Static $J(q)$ Calculation- - Heisenberg Model}

We derive the linear response to an external magnetic field $\mathbf{B}$ for the Heisenberg model, whose Hamiltonian is given as

$$
\mathcal{H}=-\sum_{\mathbf{T} a} \sum_{\mathbf{T}^{\prime} a^{\prime}} J_{\mathbf{T} a \mathbf{T}^{\prime} a^{\prime}} \mathbf{S}_{\mathbf{T} a} \cdot \mathbf{S}_{\mathbf{T}^{\prime} a^{\prime}}+g \mu_{B} \sum_{\mathbf{T} a} \mathbf{S}_{\mathbf{T} a} \cdot \mathbf{B}_{\mathbf{T} a}
$$

where $\mathbf{S}_{\mathbf{T} a}$ is the spin at $\mathbf{T} a$ ( $\mathbf{T}$ is for primitive cell, $a$ specify magnetic site in a cell). $J_{\mathbf{T} a \mathbf{T} a}=0 . \quad J_{\mathbf{T} a \mathbf{T}^{\prime} a^{\prime}}=J_{\mathbf{T}^{\prime} a^{\prime} \mathbf{T} a}$. The equation of motion $-i \hbar \dot{\mathbf{S}}_{\mathbf{T} a}=\left[\mathcal{H}, \mathbf{S}_{\mathbf{T} a}\right]$ is written as

$$
\hbar \dot{\mathbf{S}}_{\mathbf{T} a}=\mathbf{S}_{\mathbf{T} a} \times\left(2 \sum_{\mathbf{T}^{\prime} a^{\prime}} J_{\mathbf{T} a \mathbf{T}^{\prime} a^{\prime}} \mathbf{S}_{\mathbf{T}^{\prime} a^{\prime}}-g \mu_{B} \mathbf{B}_{\mathbf{T} a}\right)
$$

We introduce $g \mu_{B} \mathbf{B}=2 \mathbf{b}$, and $\mathbf{S}_{\mathbf{T} a}=\mathbf{S}_{\mathbf{T} a}^{0}+\Delta \mathbf{S}_{\mathbf{T} a} . \mathbf{S}_{\mathbf{T} a}^{0}$ is the static spin configuration. Then Eq. (18) reduces to

$$
\begin{array}{r}
\hbar \Delta \mathbf{S}_{\mathbf{T} a}=\mathbf{S}_{\mathbf{T} a}^{0} \times\left(2 \sum_{\mathbf{T}^{\prime} a^{\prime}} J_{\mathbf{T} a \mathbf{T}^{\prime} a^{\prime}} \Delta \mathbf{S}_{\mathbf{T}^{\prime} a^{\prime}}\right)+\Delta \mathbf{S}_{\mathbf{T} a} \times\left(2 \sum_{\mathbf{T}^{\prime} a^{\prime}} J_{\mathbf{T} a \mathbf{T}^{\prime} a^{\prime}} \mathbf{S}_{\mathbf{T}^{\prime} a^{\prime}}\right)-2 \mathbf{S}_{\mathbf{T} a}^{0} \times \mathbf{b}_{\mathbf{T} a} \\
(19)=\sum_{\mathbf{T}^{\prime} a^{\prime}}\left(2 \mathbf{S}_{\mathbf{T} a}^{0} J_{\mathbf{T} a \mathbf{T}^{\prime} a^{\prime}}\right) \times \Delta \mathbf{S}_{\mathbf{T}^{\prime} a^{\prime}}-\left(2 \sum_{\mathbf{T}^{\prime} a^{\prime}} J_{\mathbf{T} a \mathbf{T}^{\prime} a^{\prime}} \mathbf{S}_{\mathbf{T}^{\prime} a^{\prime}}^{0}\right) \times \Delta \mathbf{S}_{\mathbf{T} a}-2 \mathbf{S}_{\mathbf{T} a}^{0} \times \mathbf{b}_{\mathbf{T} a}
\end{array}
$$

Introducing the Fourier transform, $\Delta \mathbf{S}_{\mathbf{T} a}=\frac{1}{N} \sum_{\mathbf{k}} \Delta \mathbf{S}_{a}(\mathbf{k}) e^{i \mathbf{k}(\mathbf{T}+\mathbf{a})}$, Eq. (19) reduces to

$$
\hbar \Delta \mathbf{S}_{a}(\mathbf{k})=\sum_{a^{\prime}}\left(2 \mathbf{S}_{a}^{0} J_{a a^{\prime}}(\mathbf{k})-\left(2 \sum_{a^{\prime \prime}} J_{a a^{\prime \prime}}(0) \mathbf{S}_{a^{\prime \prime}}^{0}\right) \delta_{a a^{\prime}}\right) \times \Delta \mathbf{S}_{a^{\prime}}(\mathbf{k})-2 \mathbf{S}_{a}^{0} \times \mathbf{b}_{a}(\mathbf{k}) .
$$

Assuming $\Delta \mathbf{S}_{a}(\mathbf{k}) \propto e^{-i \omega t / \hbar}$, we have

$$
\sum_{a^{\prime}}\left(\frac{i \omega \delta_{a a^{\prime}}}{2}+\mathbf{S}_{a}^{0} J_{a a^{\prime}}(\mathbf{k})-\left(\sum_{a^{\prime \prime}} J_{a a^{\prime \prime}}(0) \mathbf{S}_{a^{\prime \prime}}^{0}\right) \delta_{a a^{\prime}}\right) \times \Delta \mathbf{S}_{a^{\prime}}(\mathbf{k})=\mathbf{S}_{a}^{0} \times \mathbf{b}_{a}(\mathbf{k}) .
$$

Let us consider the collinear ground state. Then $\mathbf{S}_{a}^{0}=S_{a} \mathbf{e}_{z}\left(S_{a}\right.$ is the size of spin, including sign). We have

$$
\sum_{a^{\prime}}\left(\frac{i \omega \delta_{g a^{\prime}}}{2 S_{a}^{\prime}}\right) \Delta \mathbf{S}_{a^{\prime}}(\mathbf{k})+\sum_{a^{\prime}}\left(J_{a a^{\prime}}(\mathbf{k})-\left(\sum_{a^{\prime \prime}} \frac{1}{S_{a}} J_{a a^{\prime \prime}}(0) S_{a^{\prime \prime}}\right) \delta_{a a^{\prime}}\right) \mathbf{e}_{z} \times \Delta \mathbf{S}_{a^{\prime}}(\mathbf{k})=\mathbf{e}_{z} \times \mathbf{b}_{a}(\mathbf{k}) .
$$

Using $\mathbf{S}=S^{+} \frac{\mathbf{e}_{x}-i \mathbf{e}_{y}}{2}+S^{-} \frac{\mathbf{e}_{x}+i \mathbf{e}_{y}}{2}+S^{z} \mathbf{e}_{z}$, and $\mathbf{e}_{z} \times\left(\mathbf{e}_{x} \pm i \mathbf{e}_{y}\right)=\mp i\left(\mathbf{e}_{x} \pm i \mathbf{e}_{y}\right)$ we have,

$$
\begin{aligned}
& \sum_{a^{\prime}}\left(\frac{\omega \delta_{a a^{\prime}}}{2 S_{a}}-\bar{J}_{a a^{\prime}}(\mathbf{k})\right) S_{a^{\prime}}^{+}(\mathbf{k})=b_{a}^{+}(\mathbf{k}) . \\
& \sum_{a^{\prime}}\left(\frac{\omega \delta_{a a^{\prime}}}{2 S_{a}}+\bar{J}_{a a^{\prime}}(\mathbf{k})\right) S_{a^{\prime}}^{-}(\mathbf{k})=b_{a}^{-}(\mathbf{k}),
\end{aligned}
$$

where

$$
\bar{J}_{a a^{\prime}}(\mathbf{k})=J_{a a^{\prime}}(\mathbf{k})-\left(\sum_{a^{\prime \prime}} \frac{1}{S_{a}} J_{a a^{\prime \prime}}(0) S_{a^{\prime \prime}}\right) \delta_{a a^{\prime}}
$$

Only the difference between $\bar{J}_{a a^{\prime}}(\mathbf{k})$ and $J_{a a^{\prime}}(\mathbf{k})$ are diagonal parts. These are determined so that $\int d^{3} k J_{a a}(\mathbf{k})=0$. Eq. (23) is the same as Eq. (14). 


\section{REFERENCES}

[1] T. Moriya, Y. Takahashi, and K. Ueda. Antiferromagnetic spin fluctuations and superconductivity in 2-dimensional metals - a possible model for high-tc oxides. Journal of the Physical Society of Japan, 59(8):2905-2915, 1990. 39.

[2] A. J. Millis, H. Monien, and D. Pines. Phenomenological model of nuclear-relaxation in the normal state of yba2cu3o7. Physical Review B, 42(1):167-178, 1990. 30 Part A.

[3] G. G. Lonzarich and L. Taillefer. Effect of spin fluctuations on the magnetic equation of state of ferromagnetic or nearly ferromagnetic metals. J. Phys. C: Solid State Phys., 18:4339, 1985.

[4] S. N. Kaul. Spin-fluctuation theory for weak itinerant-electron ferromagnets: revisited. J. Phys: Cond. Matter, 11:7597, 1999.

[5] Xiangang Wan, Quan Yin, and Sergej Y. Savrasov. Calculation of magnetic exchange interactions in mott-hubbard systems. Phys. Rev. Lett., 97(26):266403, 2006.

[6] I. V. Solovyev and K. Terakura. Effective single-particle potentials for mno in light of interatomic magnetic interactions: Existing theories and perspectives. Phys. Rev. B, 58(23):15496-15507, Dec 1998.

[7] T. Kotani. Optimized effective potential method for solids with exact exchange and exact RPA correlation. J.Phys.: Condens. Matter, 10:9241, 1998.

[8] J. Muscat, A. Wander, and N. M. Harrison. On the prediction of band gaps from hybrid functional theory. Chemical Physics Letters, 342(3-4):397-401, 2001. 33.

[9] Axel D. Becke. Density-functional thermochemistry. iii. the role of exact exchange. The Journal of Chemical Physics, 98(7):5648-5652, 1993.

[10] C. Franchini, V. Bayer, R. Podloucky, J. Paier, and G. Kresse. Density functional theory study of mno by a hybrid functional approach. Phys. Rev. B, 72(4):045132, 2005.

[11] John P. Perdew, Kieron Burke, and Matthias Ernzerhof. Generalized gradient approximation made simple. Phys. Rev. Lett., 77(18):3865-3868, Oct 1996.

[12] Ibério de P. R. Moreira, Francesc Illas, and Richard L. Martin. Effect of fock exchange on the electronic structure and magnetic coupling in nio. Phys. Rev. B, 65(15):155102, Mar 2002.

[13] F. Fuchs, J. Furthmuller, F. Bechstedt, M. Shishkin, and G. Kresse. Quasiparticle band structure based on a generalized kohn-sham scheme. Physical Review B, 76(11):115109, 2007.

[14] Joachim Paier, Martijn Marsman, and Georg Kresse. Why does the b3lyp hybrid functional fail for metals? The Journal of Chemical Physics, 127(2):024103, 2007.

[15] S. V. Faleev, M. van Schilfgaarde, and Takao Kotani. All-electron self-consistent GW approximation: Application to Si, MnO, and NiO. Phys. Rev. Lett., 93:126406, 2004.

[16] A. N. Chantis, M. van Schilfgaarde, and T. Kotani. Ab initio prediction of conduction band spin splitting in zinc blende semiconductors. Phys. Rev. Lett., 96:086405, 2006.

[17] M. van Schilfgaarde, Takao Kotani, and S. Faleev. Quasiparticle self-consistent gw theory. Phys. Rev. Lett., 96:226402, 2006.

[18] Athanasios N. Chantis, Mark van Schilfgaarde, and Takao Kotani. Quasiparticle self-consistent gw method applied to localized 4f electron systems. Physical Review B, 76(16):165126, 2007.

[19] Takao Kotani, Mark van Schilfgaarde, and Sergey V. Faleev. Quasiparticle self-consistent gw method: A basis for the independent-particle approximation. Physical Review B, 76(16):165106, 2007.

[20] Fabien Bruneval, Nathalie Vast, Lucia Reining, M. Izquierdo, F. Sirotti, and N. Barrett. Exchange and correlation effects in electronic excitations of cu[sub 2]o. Physical Review Letters, 97(26):267601, 2006.

[21] M. Shishkin, M. Marsman, and G. Kresse. Accurate quasiparticle spectra from self-consistent gw calculations with vertex corrections. Physical Review Letters, 99(24):246403, 2007.

[22] Francesco Sottile, Valerio Olevano, and Lucia Reining. Parameter-free calculation of response functions in time-dependent density-functional theory. Phys. Rev. Lett., 91(5):056402, Jul 2003.

[23] K. Terakura, A. R. Williams, T. Oguchi, and J. Kübler. Transition-metal monoxides: Band or mott insulators. Phys. Rev. Lett., 52(20):1830-1833, May 1984.

[24] M. Tanaka et al. Molecular-beam epitaxy of mnas thin-films on gaas. Jour. Vac. Sci. Tech. B, 12:10911094, 1994.

[25] J. Kunes, V. I. Anisimov, S. L. Skornyakov, A. V. Lukoyanov, and D. Vollhardt. Nio: Correlated band structure of a charge-transfer insulator. Physical Review Letters, 99(15):156404, 2007.

[26] A. G. Petukhov, I. I. Mazin, L. Chioncel, and A. I. Liechtenstein. Correlated metals and the lda+u method. Phys. Rev. B, 67:153106, 2003.

[27] J. H. Shim, K. Haule, and G. Kotliar. Fluctuating valence in a correlated solid and the anomalous properties of delta-plutonium. NATURE, 446, 2007. 
[28] Atsushi Fujimori and Fujio Minami. Valence-band photoemission and optical absorption in nickel compounds. Phys. Rev. B, 30(2):957-971, Jul 1984.

[29] H. Akai and P. H. Dederichs. Local moment disorder in ferromagnetic alloys. Phys. Rev. B, 47(14):87398747, Apr 1993.

[30] J. W. D. Connolly and A. R. Williams. Phys. Rev. B, 27:5169, 1983.

[31] Ivan Rungger and Stefano Sanvito. Ab initio study of the magnetostructural properties of mnas. Phys. Rev. B, 74(2):024429, Jul 2006.

[32] L. M. Sandratskii and E. Sasioglu. Unusual sequence of phase transitions in mnas: First-principles study. Phys. Rev B, 74:214422, 2006.

[33] T. Oguchi, K. Terakura, and A. R. Williams. Band theory of the magnetic interaction in mno, mns, and nio. Phys. Rev. B, 28(11):6443-6452, Dec 1983.

[34] A. I. Liechtenstein, M. I. Katsnelson, V. P. Antropov, and V. A. Gubanov. J. Magn. Magn. Mater., 67:65, 1987.

[35] J. F. Cooke, J. W. Lynn, and H. L. Davis. Calculations of the dynamic susceptibility of nickel and iron. Phys. Rev. B, 21(9):4118-4131, May 1980.

[36] S. Y. Savrasov. Linear response calculations of spin fluctuations. Phys. Rev. Lett., 81(12):2570-2573, Sep 1998.

[37] K. Karlsson and F. Aryasetiawan. Spin-wave excitation spectra of nickel and iron. Phys. Rev. B, 62(5):3006-3009, Aug 2000.

[38] V. P. Antropov. J. Mag. Mag. Mat., 262:L192, 2003.

[39] M. I. Katsnelson and A. I. Lichtenstein. Magnetic susceptibility, exchange interactions and spin-wave spectra in the local spin density approximation. Journal of Physics-Condensed Matter, 16(41):7439-7446, 2004.

[40] P. W. Anderson and H. Hasegawa. Considerations on double exchange. Phys. Rev., 100(2):675-681, Oct 1955.

[41] Mark van Schilfgaarde and O. N. Mryasov. Anomalous exchange interactions in iii-v dilute magnetic semiconductors. Phys. Rev. B, 63(23):233205, May 2001.

[42] Y. Kohgi, I. Ishikawa, and Y Endoh. Solid State Communication, 11:391, 1972.

[43] M. T. Hutchings and E. J. Samuelsen. Measurement of spin-wave dispersion in nio by inelastic neutron scattering and its relation to magnetic properties. Phys. Rev. B, 6(9):3447-3461, Nov 1972.

[44] J. B. Goodenough and J. A. Kafalas. High-pressure study of the first-order phase transition in mnas. Phys. Rev., 157(2):389-395, May 1967.

[45] W. L. Roth. Magnetic structures of mno, feo, coo, and nio. Phys. Rev., 110(6):1333-1341, Jun 1958.

[46] J. L. Xu, M. van Schilfgaarde, and G. D. Samolyuk. Role of disorder in mn:gaas, cr:gaas, and cr:gan. Phys. Rev. Lett., 94:097201, 2005.

[47] R. Y. Gu and V. P. Antropov. Quantum spin effect and short-range order above the curie temperature. Preprint http://arxiv.org/abs/cond-mat/0508781. 


\section{Low Energy, Coherent, Stoner-like Excitations in $\mathrm{CAFE}_{2} \mathrm{As}_{2}$}

Magnetic interactions are likely to play a key role in mediating superconductivity in the recently discovered family of iron pnictides; however, their character is not yet well understood $[1,2,3]$. In particular, whether the system is best described in terms of large, local magnetic moments centered at each Fe site, in which case elementary excitations are collective spin waves (SWs) or are itinerant (elementary excitations characterized by single-particle electronhole transitions) is a subject of great debate. This classification also depends on the energy scale of interest. The most relevant energy scale in $\mathrm{CaFe}_{2} \mathrm{As}_{2}$ and other pnictides ranges to about twice the Néel temperature, i.e. $2 T_{N} \approx 40 \mathrm{meV}$. Unfortunately, neutron scattering experiments have focused on the character of excitations in the 150-200 meV range [4,5], much larger than energy that stabilizes observed magnetism or superconductivity. Experiments in Refs. $[4,5]$, while very similar, take completely different points of view concerning the magnetic excitations that they observe. There is a similar dichotomy in theoretical analyses of magnetic interactions [6, 7]. Model descriptions usually postulate a local-moments picture [8]. Most $a b$ initio studies start from the local spin-density approximation (LSDA) to density functional theory. While the LSDA traditionally favors itinerant magnetism (weak on-site Coulomb correlations), practitioners strongly disagree about the character of pnictides; indeed the same results are used as a proof of both localized and itinerant descriptions $[7,6]$.

The dynamic magnetic susceptibility (DMS) is the central quantity that uniquely characterizes magnetic excitations. It can elucidate the origins of magnetic interactions and distinguish between localized and itinerant character. However, it is difficult to compute in practice; studies to date have been limited to a few simple systems. Here we adapt an all-electron linear response technique developed recently $[9,10]$ to calculate the transverse DMS, $\chi(\mathbf{q}, \omega)$. Aside from SWs seen in neutron scattering, we find low energy particle-hole excitations at low $q$, and also at high $q$. In stark contrast to conventional particle-hole excitations in the Stoner continuum, they can be sharply peaked in energy (resonances) and can be measured.

The full transverse DMS $\chi\left(\mathbf{r}, \mathbf{r}^{\prime}, \mathbf{q}, \omega\right)$ is a function of coordinates $\mathbf{r}$ and $\mathbf{r}^{\prime}$ (confined to the unit cell). It is obtained from the non-interacting susceptibility $\chi_{0}\left(\mathbf{r}, \mathbf{r}^{\prime}, \mathbf{q}, \omega\right)$ via the standard relation $[11]$

$$
\chi=\chi_{0}\left[1-\chi_{0} I\right]^{-1}
$$

where $I$ is the exchange-correlation kernel. When computed within the time-dependent LSDA (TDLDA) $I$ is local: $I=I(\mathbf{r}) \delta\left(\mathbf{r}-\mathbf{r}^{\prime}\right)$ [11]. The $\chi_{0}$ can be obtained from the band structure using the all-electron methodology we developed $[9,10]$. Im $\chi_{0}$ originates from spin-flip transitions between occupied states at $\mathbf{k}$ and unoccupied states at $\mathbf{k}+\mathbf{q}$ : it is a $k$-resolved joint density of states $D$ decorated by products $P$ of four wave functions [10]

$$
\begin{array}{r}
D(\mathbf{k}, \mathbf{q}, \omega)=f\left(\epsilon_{\mathbf{k}}^{\uparrow}\right)\left(1-f\left(\epsilon_{\mathbf{q}+\mathbf{k}}^{\downarrow}\right)\right) \delta\left(\omega-\epsilon_{\mathbf{q}+\mathbf{k}}^{\downarrow}+\epsilon_{\mathbf{k}}^{\uparrow}\right) \\
\operatorname{Im} \chi_{0}^{+-}=\int d \omega d^{3} \mathbf{k} P\left(\mathbf{r}, \mathbf{r}^{\prime}, \mathbf{k}, \mathbf{q}\right) \times D(\mathbf{k}, \mathbf{q}, \omega)
\end{array}
$$

Because of the computational burden Eq. (3) poses, we make a simplification, mapping $\chi_{0}$ onto the local magnetization density which is assumed to rotate rigidly. The full $\chi_{0}\left(\mathbf{r}, \mathbf{r}^{\prime}, \mathbf{q}, \omega\right)$ simplifies to the discrete matrix $\chi_{0}\left(\mathbf{R}, \mathbf{R}^{\prime}, \mathbf{q}, \omega\right)$ associated with pairs of magnetic sites $\left(\mathbf{R}, \mathbf{R}^{\prime}\right)$ in the unit cell; and $I(\mathbf{r})$ simplifies to a diagonal matrix $I_{\mathbf{R R}}$. In Ref. [10] we show that we need not compute $I$ explicitly but can determine it from a sum rule. $I$ can be identified with the Stoner parameter in models. We essentially follow the procedure described in detail in Ref. [10], and obtain $\chi(\mathbf{q}, \omega)$ as a $4 \times 4$ matrix corresponding to the four Fe sites in the unit 


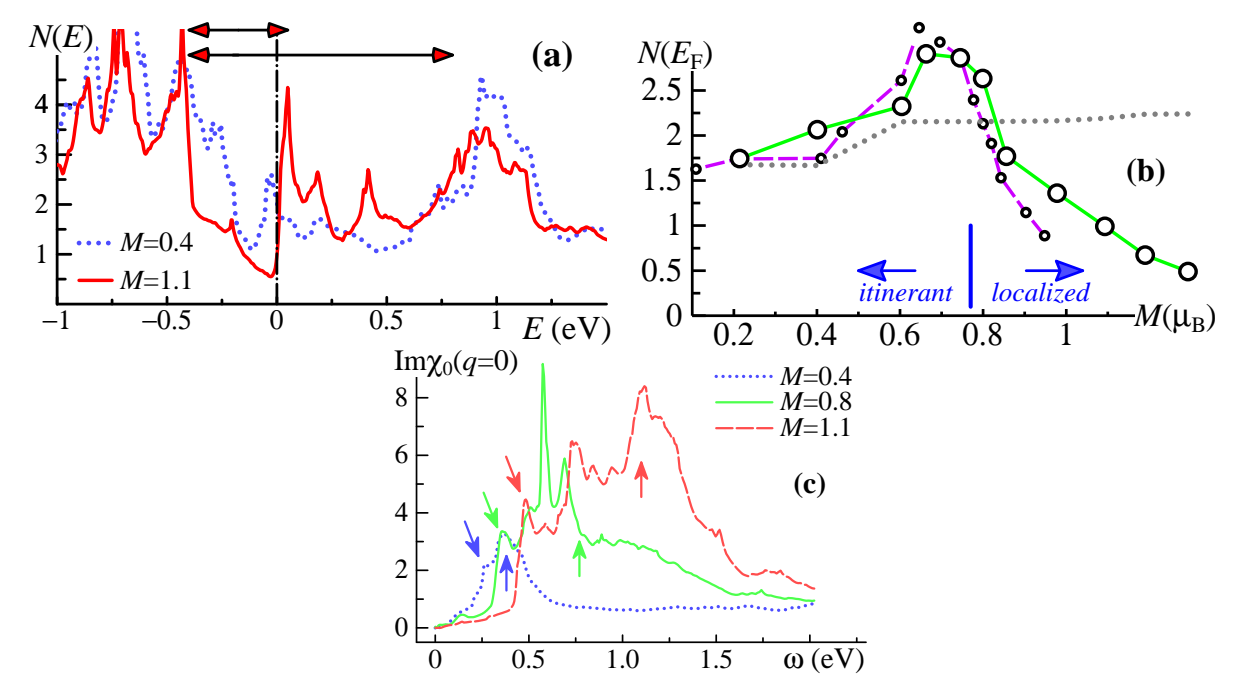

Figure 1. (Color online) (a) $N(E)$, in units of $\mathrm{eV}^{-1}$ per cell containing one Fe atom. Data are shown for $M=0.4 \mu_{B}$ and $1.1 \mu_{B}$. Both kinds of transitions are reflected in peaks in $\chi_{0}(q=0, \omega)$, shown in panel (c) for $M=0.4,0.8$, and $1.1 \mu_{B}$. (b) $N\left(E_{F}\right)$ as a function of moment $M$. The blue vertical bar denotes the experimental moment. It also approximately demarcates the transition from itinerant to localized behavior. (c) bare susceptibility $\chi_{0}(q=0, \omega)$ in the same units. The text discusses the significance of the arrows in panels (a) and (c).

cell. For Fe and Ni these approximations yield results in rather good agreement with the full TDLDA results. To make connection with neutron experiments, spectra are obtained from the matrix element $\sum_{\mathbf{R}, \mathbf{R}^{\prime}}<e^{i \mathbf{q} \cdot \mathbf{R}}\left|\chi\left(\mathbf{R}, \mathbf{R}^{\prime}, \mathbf{q}, \omega\right)\right| e^{i \mathbf{q} \cdot \mathbf{R}^{\prime}}>$. For brevity we omit indices $\mathbf{R R}^{\prime}$ henceforth.

We analyze the low-temperature (striped) phase of $\mathrm{CaFe}_{2} \mathrm{As}_{2}$ within the LSDA, and show how these states depend critically on the Zeeman field generated by the Fe magnetic moment $M$. We do this by varying the Fe-As bond length $R_{\mathrm{Fe}-\mathrm{As}}$ : its main effect is to control the magnetic moment $M$, which in turn strongly affects the character of magnetic interaction. Similar changes can be accomplished by other means (e.g. with the external $B$ field), as we will report elsewhere. Results for tetragonal and orthorhombic striped structures are very similar, suggesting that the slight difference in measured $a$ and $b$ lattice parameters plays a minor role in the magnetic structure. Experimental lattice parameters were employed[12].

In Fig. 1(a), the density of states $N(E)$ is shown over a $2 \mathrm{eV}$ energy window for a small and large moment case $\left(M=0.4 \mu_{B}\right.$ and $\left.1.1 \mu_{B}\right)$. Particularly of note is a sharp peak near $E_{F}$, of width $\sim 50 \mathrm{meV}$. This narrow band is found to consist almost entirely of majority-spin $\mathrm{Fe}$ $d_{x y}$ and $d_{y z}$ orbitals. The peak falls slightly below $E_{F}$ for $M=0.4 \mu_{B}$ and slightly above for $M=1.1 \mu_{B}$. Thus, as $R_{\mathrm{Fe}-\mathrm{As}}$ is smoothly varied so that $M$ changes continuously, this peak passes through $E_{F}$. As a result $N\left(E_{F}\right)$ reaches a maximum around $M=0.8 \mu_{B}$ (Fig. $1(b)$ ). The fact that this unusual dependence originates in the magnetic part of the Hamiltonian can be verified by repeating the calculation in the nonmagnetic case. As Fig. $1(b)$ shows, the nonmagnetic $N\left(E_{F}\right)$ is large, and approximately independent of $R_{\mathrm{Fe}-\mathrm{As}}$. In summary, the magnetic splitting produces a pseudogap in $N(E)$ for large $M$; the pseudogap shrinks as $M$ decreases and causes a narrow band of Fe $d$ states to pass through $E_{F}$, creating a 
sharp maximum in $N\left(E_{F}\right)$ near $M=0.8 \mu_{B}$. As we will show in the following, this moment demarcates a point of transition from itinerant to localized behavior (see also Ref. [13]).

Next we turn to magnetic excitations. In the standard picture of magnons, $\operatorname{Im} \chi_{0}(\omega)$ is significant only for frequencies exceeding the magnetic (Stoner) splitting of the $d$ bands, $\epsilon_{d}^{\downarrow}-\epsilon_{d}^{\uparrow}=I M$ (cf Eq. 2). In such cases $\operatorname{Im} \chi_{0}$ is small at low $(q, \omega)$ and well defined magnons appear at energies near $\left|1-I \operatorname{Re} \chi_{0}\right|=0$ (cf Eq. 1). As $\operatorname{Im} \chi_{0}$ increases the SW spectrum $\bar{\omega}(\mathbf{q})$ broadens; as $\operatorname{Im} \chi_{0}$ becomes large the spectrum can become incoherent, or (Stoner) peaks can arise from $\operatorname{Im} \chi_{0}$, possibly enhanced by small $1-I \operatorname{Re} \chi_{0}$. Figure $1(c)$ shows $\operatorname{Im} \chi_{0}(q=0, \omega)$ on a broad energy scale. The description of $N(E)$ leads to a classification of Stoner transitions into three main types.

(1) Excitations from the usual Stoner splitting. In a rigid-band model, no bands are spinsplit but the $d$ states, which are split by $I M$. Only they contribute to $\operatorname{Im} \chi_{0}(q=0, \omega)$ because all other pairs of states are spatially orthogonal and their cell average vanishes. The vertical arrows in Fig. $1(c)$ demarcate $I M$, assuming $I \approx 1 \mathrm{eV}$ known to apply to $3 d$ transition metals. Peaks which approximately correspond to $I M$ we identify with the usual Stoner splitting. The splitting can be seen in the density of states (DOS), Fig. 1 (a) for $M=1.1 \mu_{B}$; it is indicated by a large red arrow showing transitions between Fe states (largely $d_{x z}$ and $d_{x y}$ ) near $-0.4 \mathrm{eV}$ to unoccupied $d$ states below $1 \mathrm{eV}$.

(2) Transitions to states of $\left(d_{x y}, d_{y z}, d_{x z}\right)$ character just above the psueudogap, depicted by a small red arrow in Fig. 1(a), and slanting arrows in Fig. 1(d). These are the excitations probably detected in neutron measurements $[4,5]$. This pseudogap is well defined for $M \geq 1.1 \mu_{B}$, but is modified as $M$ decreases, which leads to the following:

(3) Near $M=0.8 \mu_{B}$, the narrow $d$ band passes through $E_{F}$, opening up channels, not previously considered, for low-energy, particle-hole transitions within this band. When $M$ reaches $0.4 \mu_{B}$, this band has mostly passed through $E_{F}$ and the pseudogap practically disappears.

What makes the pnictide systems so unusual is that $\operatorname{Im} \chi_{0}$ is already large at very low energies $(\sim 10 \mathrm{meV})$ once the sharp peak in $N(E)$ approaches $E_{F}$. One of our central findings is that this system undergoes a transition from localized to a coexistence of localized and itinerant behavior as $M$ decreases from $M \gtrsim 1.1 \mu_{B}$ to $M \approx 0.8 \mu_{B}$. Moreover, the itinerant character is of an unusual type: elementary excitations are mostly single particle-hole like: they can be well defined in energy and $q$. Those represent coherent excitations. The dependence of $N\left(E_{F}\right)$ on $M$ is not only responsible for them, but also may explain the unusual linear temperaturedependence of paramagnetic susceptibility, and the appearance of a Lifshitz transition with Co doping [14].

Figure 2 focuses on the antiferromagnetic (AFM) line, $\mathbf{q}=[H 00] 2 \pi / a$. Panel (a) shows the full $\operatorname{Im} \chi(\omega)$ for $M=1.1 \mu_{B}$ for several $q$-points spanning the entire line, $0<H<1$. At low $q$, peaks $\bar{\omega}$ in $\chi$ are sharp; and $\bar{\omega}$ depends on $H$ in the expected manner $(\bar{\omega} \propto H)$. $\bar{\omega}$ reaches a maximum near $H=1 / 2$ (Figure $2(b)$ ), for all three values of $M$. The peaks broaden with increasing $q$; nevertheless we can associate them with magnons, because they coincide closely with vanishing $\left|1-I \operatorname{Re} \chi_{0}\right|$ and $\Gamma$ is not too large. The magnon character is preserved for most $q$ at all moments, as Figure $2(b)$ shows: but for $H>0.8$ the peaks are strongly broadened, especially for small $M . \bar{\omega}$ is in good agreement with neutron data of Ref.[5], except that neutron data are apparently smaller than large $M$ calculations predict [15].

One experimental measure of the validity of the local-moment picture is the ratio of halfwidth at half-maximum $\Gamma$ to $\bar{\omega}$, shown in Figure $2(d)$. For large $M$ and most of $q, \Gamma / \bar{\omega} \sim 0.15$ 0.18 . For intermediate and small $M, \Gamma / \bar{\omega} \geq 0.2$ for a wide diapason of $q$. This is significant: it reflects the increasing Stoner character of the elementary excitations. Were there an abrupt transition into a conventional Stoner continuum as argued in Ref. [5], it would be marked by 

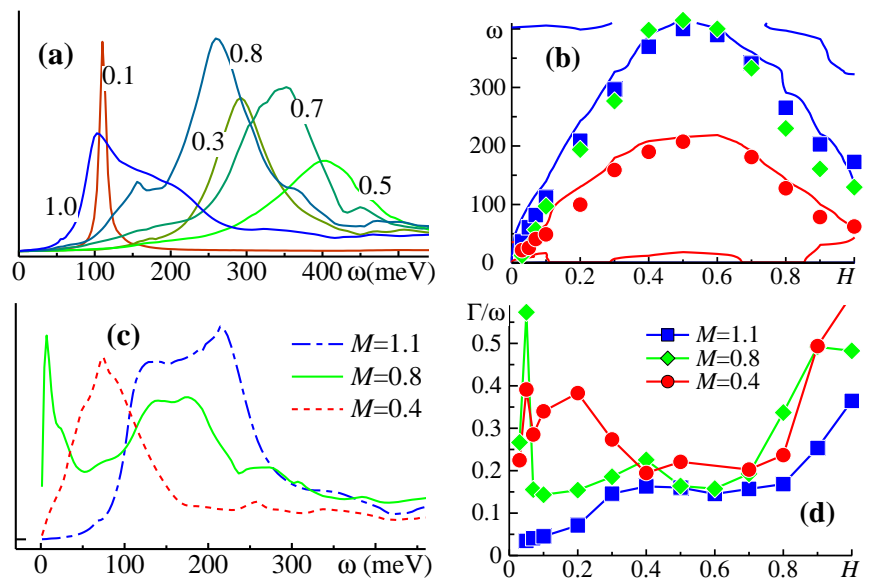

Figure 2. (Color online) $\operatorname{Im} \chi(\mathbf{q}, \omega)$ along the AFM axis, $\mathbf{q}=[H, 0,0] 2 \pi / a$. (a) $\operatorname{Im} \chi(\omega)$ for various $H$ and $M=1.1 \mu_{B}$. Extracted from $\operatorname{Im} \chi(H, \omega)$ are peak positions $\bar{\omega}(H)(\mathrm{meV})$ and half-width at half-maximum $\Gamma$. (b) and (d) depict $\bar{\omega}$ and the ratio $\Gamma / \bar{\omega}$, for the three moments shown in the key. Panel (b) also depicts, as solid lines, contours $\left|1-I \operatorname{Re} \chi_{0}\right|=0$ in the $(\omega, H)$ plane, for $M=1.1$ and $0.4 \mu_{B}$. Panel $(\mathbf{c})$ shows $\operatorname{Im} \chi(H=0.9, \omega)$ for the three moments.

an abrupt change in $\Gamma / \bar{\omega}$. This is not observed; yet damping appears to increase with energy and $q$, reflecting normal metallic behavior.

Spectra for $H=0.9$ (Figure $2(c)$ ) adumbrate two important findings of this work. When $M=0.8 \mu_{B}$, a sharp peak in $\chi(\omega)$ appears near $10 \mathrm{meV}$. There is a sharp peak in $\chi_{0}(\omega)$ at $\bar{\omega} \approx 10 \mathrm{meV}$ also, classifying this as a particle-hole excitation originating from the narrow $d$ band depicted in Figure 1. Being well defined in energy it is coherent, analogous to a SW, only with a much larger $\Gamma / \bar{\omega}$. Yet it is strongly enhanced by collective interactions, since $\left|1-I \operatorname{Re} \chi_{0}\right|$ ranges between 0.1 and 0.2 for $\omega<100 \mathrm{meV}$. This new kind of itinerant excitation will be seen at many values of $q$, typically at small $q$. The reader many note the sharp rise and fall in $\Gamma / \bar{\omega}$ at small $q$ in Figure $2(d)$. This anomaly reflects a point where a SW and a particle-hole excitation coalesce to the same $\bar{\omega}$.

Returning to $H=0.9$ when $M=1.1$, there is a standard (broadened) $\mathrm{SW}$ at $200 \mathrm{meV}$; $c f$ contours in Figure 2(b). A second, low-energy excitation can be resolved near $120 \mathrm{meV}$. This is no corresponding zero in the denominator in Eq. (1), but no strong peak in $\chi_{0}(\omega)$, either. This excitation must be classified as a hybrid intermediate between Stoner excitations and SWs. Only a single peak remains when $M=0.4 \mu_{B}$ and the peak in $N(E)$ has mostly passed through $E_{F}$ (Figure 1).

Along the ferromagnetic $(\mathrm{FM})$ line $\mathbf{q}=(0, K, 0) 2 \pi / a, \chi(\omega)$ is more complex, and more difficult to interpret. At $M=1.1 \mu_{B}$ sharp, well defined collective excitations are found at low $q$, and broaden with increasing $q$. There is a reasonably close correspondence with the zeros of $\left|1-I \operatorname{Re} \chi_{0}\right|$ and peaks in $\chi$, as Figure $3(b)$ shows. The $M=0.8 \mu_{B}$ case is roughly similar, except that for $K>0.4$ excitations cannot be described by a single peak. Note that for fixed $q$, propagating spin fluctuations (characterized by peaks at $\bar{\omega}$ ) can exist at multiple energies - with the magnetic analog of the dielectric function passing through zero and sustaining plasmons at multiple energies. Peaks in $\chi(\omega)$ can broaden as a consequence of this; the $K=7 / 12,9 / 12$ and $11 / 12$ data of Fig. $3(a)$ are broadened in part by this mechanism as distinct from the usual one (intermixing of Stoner excitations). Second, consider how $\partial \bar{\omega} / \partial q$ 

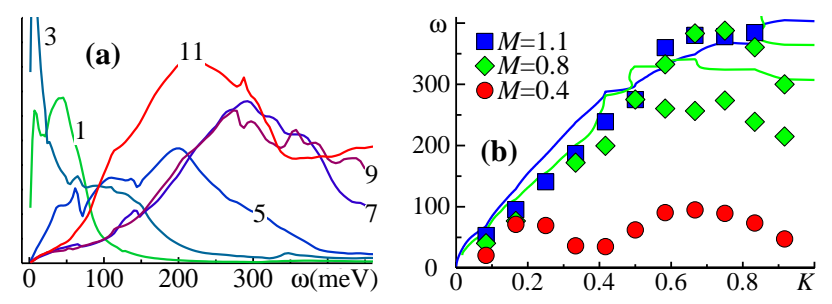

Figure 3. (Color online) $\operatorname{Im} \chi(\mathbf{q}, \omega)$ along the FM axis, $\mathbf{q}=[0, K, 0] 2 \pi / a$. (a) $\operatorname{Im} \chi(\omega)$ at $K=1 / 12,3 / 12,5 / 12,7 / 12,9 / 12,11 / 12$, for $M=0.8 \mu_{B}$. Strong Stoner excitations can be seen for $K=1 / 12$ and $3 / 12$ near $\bar{\omega} \sim 10-20 \mathrm{meV}$. (b): Contours $\left|1-I \operatorname{Re} \chi_{0}\right|=0$ in the $(\omega, K)$ plane, for $M=1.1 \mu_{B}$ and $0.8 \mu_{B}$, analogous to Figure $2(b)$, and dominant peak positions $\bar{\omega}$ obtained by a nonlinear least-squares fit of one or two Gaussian functions to $\chi(\omega)$ over the region where peaks occur.

changes with $M$ for $K>0.5$ (Fig. 3(b)). When $M=1.1 \mu_{B}, \bar{\omega}$ increases monotonically with $K$. For $M=0.8 \mu_{B}, \bar{\omega}$ has a complex structure but apparently reaches a maximum before $K$ reaches 1 . The fact that $\partial \bar{\omega} / \partial q$ changes sign is significant: it marks the disappearance of magnetic frustration between the ferromagnetically aligned spins at low moments, and the emergence of stable FM order along [010], characteristic of local-moment behavior (see also Ref.[16]). Experimentally, Ref. [5] reports $\partial \bar{\omega} / \partial q>0$ for $K>0.5$. While our calculations provide a clear physical interpretation, we note significant differences in the moment where we observe this effect $\left(M=0.8-1.1 \mu_{B}\right)$ and the effective spin $(S=0.2)$ used in Ref.[5]. Finally, we find that the collective mode frequencies (spin wave spectrum) only weakly depend on $M$, even for large $M$.

Figure 4. (Color online) $\operatorname{Im} \chi(\mathbf{q}, \omega)$ along the $c$ axis, $\mathbf{q}=[0,0, L] 2 \pi / c$. (a) $\operatorname{Im} \chi(\omega)$ at values of $L$ listed in the key, for $M=0.8 \mu_{B}$. When $M=1.1 \mu_{B}$ (not shown) the spectrum is well characterized by a single sharp peak at any $L$. When $M=0.8$ or $0.4 \mu_{B}$ the SW peak remains, but a low-energy Stoner excitation coexists with it. The latter are most pronounced for larger $L$ but can be resolved at every $L$. (b) Peak positions (single for $M=1.1 \mu_{B}$, double for $M=0.8$ and $\left.0.4 \mu_{B}\right)$.
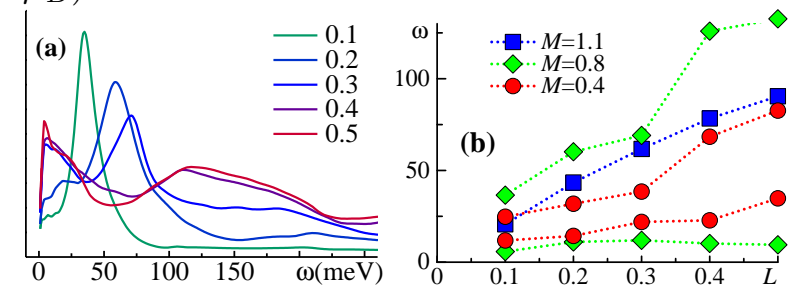

Elementary excitations along the $c$ axis, $\mathbf{q}=(0,0, L) 2 \pi / c$, bring into highest relief the transition from pure local-moment behavior (collective excitations), to one where coherent itinerant Stoner and collective excitations coexist. Collective excitations are found for all $L$ and all $M$. For $M=1.1 \mu_{B}$, a single peak is found: excitations are well described by the Heisenberg model with weak damping. Comparing Figure $4(b)$ to Figs. 2(b) and 3(b), it is apparent that $\bar{\omega}$ rises much more slowly along $L$ than along $H$ or $K$, confirming that interplane interactions are weak. When $M$ drops to $0.8 \mu_{B}$, a second-low energy peak at $\bar{\omega}^{\prime}$ emerges at energies below 
$20 \mathrm{meV}$, for small $q$ along $[0, K, 0]$, and for all $q$ along $[0,0, L]$, coexisting with the collective excitation. Why these transitions are absent for $M=1.1 \mu_{B}$ and are so strong at $M=0.8 \mu_{B}$ can be understood in terms of roughly cylindrical Fermi surface at $k=\left(1 / 2,0, k_{z}\right)$. Single spin-flip transitions between occupied states $\epsilon_{\mathbf{k}}^{\uparrow}$ and an unoccupied states $\epsilon_{\mathbf{k}+\mathbf{q}}^{\downarrow}$ separated by $\sim 10 \mathrm{meV}$ are responsible for this peak (see Eq. 2). They originate from "hot spots" where $N\left(\epsilon_{\mathrm{k}}^{\uparrow}\right)$ and $N\left(\epsilon_{\mathbf{k}+\mathbf{q}}^{\downarrow}\right)$ are both large.

From the $S W$ velocities, $(\partial \bar{\omega} / \partial q)_{q=0}$, anisotropy of the exchange couplings can be determined $[7,16,17]$. We find moderate in-plane and out-of-plane anisotropies, and predict $v_{b} / v_{a}=0.55$ and find $v_{c} / v_{a}=0.35$, where $v_{a}=490 \mathrm{meV} \cdot \AA$. Neutron scattering experiments $[17,3]$ have measured with $v_{c} / v_{a-b}$ to be $\sim 0.2-0.5$.

In summary, we broadly confirm the experimental findings of Refs. [4, 5], that there is a spectrum of magnetic excitations of the striped phase of $\mathrm{CaFe}_{2} \mathrm{As}_{2}$, which for the most part are weakly damped at small $q$ and more strongly damped at large $q$. A new kind of excitation was found, which originates from single particle-hole transitions within a narrow band of states near $E_{F}$, renormalized by a small denominator $\mid 1-I$ Re $\chi_{0} \mid$. They appear when the Fe moment falls below a threshold, at which point the narrow band passes through $E_{F}$. The character of itineracy is novel: excitations occur at low-energy, at energy scales typically below $T_{N}$, and can be sharply peaked, and thus coherent. The distinction between the two kinds of excitations is particularly observable in the anomalous dependence of $\Gamma / \bar{\omega}$ on $q$ in Figure $2(d)$. Collective spin-wave-like excitations also are unusual: multiple branches are found at some $q$. Finally, at higher $q$ we find that the SW velocity changes sign, which is also observed experimentally at similar values of $M$.

Overall, a picture emerges where localized and itinerant magnetic carriers coexist and influence each other. This description falls well outside the framework of a local-moments model such as the Heisenberg Hamiltonian. Additional low-energy neutron experiments are required to check the existence of these itinerant excitations.

\section{REFERENCES}

[1] I. I. Mazin. Nature, 464, 183 (2010)

[2] D. C. Johnson Adv. Phys. 59, 803 (2010).

[3] J. W. Lynn and P. Dai. Physica C469, 469 (2009).

[4] S. O. Diallo, V. P. Antropov, T. G. Perring, C. Broholm, J. J. Pulikkotil, N. Ni, S. L. Bud'ko, P. C. Canfield, A. Kreyssig, A. I. Goldman, and R. J. McQueeney. Phys. Rev. Lett. 102, 187206 (2009).

[5] J. Zhao, D. T. Adroja, D. Yao, R. Bewley, S. Li, X. Wang, G. Wu, X. Chen, J. Hu, P. Dai. Nature Physics 5, 555 (2009).

[6] M. D. Johannes and I. I. Mazin. Phys. Rev. B 79, 220510 (2009); I. I. Mazin and J. Schmalian. Physica C, 469, 614 (2009).

[7] Z. P. Yin, S. Lebègue, M. J. Han, B. P. Neal, S. Y. Savrasov, W. E. Pickett, Phys. Rev. Lett. 101, 047001 (2008).

[8] C. Fang, H. Yao, W. F. Tsai, J. P. Hu, and S. A. Kivelson, Phys. Rev. B 77, 224509 (2008); F. Kruger, S. Kumar, J. Zaanen and J. van den Brink. ibid. 79, 054504 (2009); C. C. Lee, W. G. Yin and W. Ku. Phys. Rev. Lett. 103, 267001 (2009).

[9] T. Kotani, M. van Schilfgaarde, S. V. Faleev, Phys. Rev. B76, 165106 (2007).

[10] T. Kotani and M. van Schilfgaarde, J. Phys. Cond. Matt. 20, 295214 (2008).

[11] R. Martin, Electronic Structure, Cambridge

[12] A. I. Goldman, D. N. Argyriou, B. Ouladdiaf, T. Chatterji, A. Kreyssig, S. Nandi, N. Ni, S. L. Bud'ko, P. C. Canfield, and R. J. McQueeney. Phys. Rev. B 78, 100506 (2008).

[13] G. D. Samolyuk and V. P. Antropov. Phys. Rev. B 79, 052505 (2009).

[14] N. Ni, A. N. Thaler, A. Kracher, J. Q. Yan, S. L. Bud'ko, P. C. Canfield. Phys. Rev. B 80, 024511 (2009), E. D. Mun, S. L. Bud'ko, N. Ni, A. N. Thaler and P. C. Canfield. Phys. Rev. B 80, 054517 (2009). 
[15] The origin of the discrepancy in $\bar{\omega}$ with experiment is not clear. It could be related to the LSDA overestimate of magnetic moment in ferropnictides.

[16] J. J. Pulikkotil1, L. Ke, M. van Schilfgaarde, T. Kotani and V. P. Antropov Supercond. Sci. Technol. 23, 054012 (2010).

[17] R. J. McQueeney, S. O. Diallo, V. P. Antropov, G. D. Samolyuk, C. Broholm, N. Ni, S. Nandi, M. Yethiraj, J. L. Zarestky, J. J. Pulikkotil, A. Kreyssig, M. D. Lumsden, B. N. Harmon, P. C. Canfield, and A. I. Goldman. Phys. Rev. Lett. 101, 227205 (2008). 